hep-ph/0112103

IFIC/01-67

\title{
Status of four-neutrino mass schemes: a global and unified approach to current neutrino oscillation data
}

\author{
M. Maltoni, 周 T. Schwetz, 吕 and J. W. F. Vallef \\ Instituto de Física Corpuscular - C.S.I.C./Universitat de València \\ Edificio Institutos de Paterna, Apt 22085, E-46071 Valencia, Spain
}

\begin{abstract}
We present a unified global analysis of neutrino oscillation data within the framework of the four-neutrino mass schemes $(3+1)$ and $(2+2)$. We include all data from solar and atmospheric neutrino experiments, as well as information from short-baseline experiments including LSND. If we combine only solar and atmospheric neutrino data, $(3+1)$ schemes are clearly preferred, whereas short-baseline data in combination with atmospheric data prefers $(2+2)$ models. When combining all data in a global analysis the $(3+1)$ mass scheme gives a slightly better fit than the $(2+2)$ case, though all four-neutrino schemes are presently acceptable. The LSND result disfavors the three-active neutrino scenario with only $\Delta m_{\mathrm{SOL}}^{2}$ and $\Delta m_{\mathrm{ATM}}^{2}$ at $99.9 \%$ C.L. with respect to the fourneutrino best fit model. We perform a detailed analysis of the goodness of fit to identify which sub-set of the data is in disagreement with the best fit solution in a given mass scheme.
\end{abstract}

PACS numbers: 14.60.P, 14.60.S, 96.40.T, 26.65, 96.60.J, 24.60

Keywords: neutrino oscillations, sterile neutrino, four-neutrino models

*Electronic address: maltoni@ific.uv.es

$\dagger$ Electronic address: schwetz@ific.uv.es

${ }_{\ddagger}^{\ddagger}$ Electronic address: valle@ific.uv.es 


\section{INTRODUCTION}

From the long-standing solar [1, 2, 3, 4, 5] and atmospheric [6, 7, 8, 9] neutrino anomalies we now have compelling evidence that an extension of the Standard Model of particle physics is necessary in the lepton sector. The most natural explanation of these experiments is provided by neutrino oscillations induced by neutrino masses and mixing with neutrino masssquared differences of the order of $\Delta m_{\mathrm{SOL}}^{2} \lesssim 10^{-4} \mathrm{eV}^{2}$ and $\Delta m_{\mathrm{ATM}}^{2} \sim 3 \times 10^{-3} \mathrm{eV}^{2}$. Explaining also the evidence of $\stackrel{(-)}{\nu}) \rightarrow \stackrel{(-)}{\nu}$ oscillations with a mass-squared difference $\Delta m_{\mathrm{LSND}}^{2} \sim 1 \mathrm{eV}^{2}$ reported by the LSND experiment [10, 11] requires an even more radical modification of the Standard Model. Currently this experiment is left out in most analyses of neutrino data. At the moment the LSND result is not confirmed nor ruled out by any other experiment, and therefore it is reasonable to see more quantitatively its impact on the physics of the lepton sector.

If all the three anomalies are explained by neutrino oscillations, and the possibility of CPT violation is neglected [12], we need at least four neutrinos to obtain the three required masssquared differences. In view of the LEP results the fourth neutrino must not couple to the Z-boson. Such a sterile neutrino with a mass in the electron-volt range has been postulated originally to provide some hot dark matter suggested by early COBE results [13, 14, 15], and after the LSND result many four-neutrino models have been proposed [16, 17, 18, 19]. A quite complete list on four-neutrino references can be found at [20].

A very important issue in the context of four-neutrino scenarios is the question of the four-neutrino mass spectrum. Two very different classes of four-neutrino mass spectra can be identified. The first class contains four types and consists of spectra where three neutrino masses are clustered together, whereas the fourth mass is separated from the cluster by the mass gap needed to reproduce the LSND result. The second class has two types where one pair of nearly degenerate masses is separated by the LSND gap from the two lightest neutrinos. These two classes are referred to as $(3+1)$ and $(2+2)$ neutrino mass spectra, respectively [21]. All possible four-neutrino mass spectra are shown in Fig. 1.

One important theoretical issue in these models is how to account for the lightness of the sterile neutrino which, ordinarily, should have mass well above the weak scale. The simplest possibility is to appeal to an underlying protecting symmetry, getting, moreover, the LSND mass at one-loop order only [13, 14]. Alternatively, the lightness of the sterile neutrino may follow from volume suppression in models based on extra dimensions [17, 18]. As for the maximal atmospheric mixing angle, it follows naturally in the models of Refs. 13, 14, 18 since to first approximation the heaviest neutrinos form a quasi-Dirac pair whose components mix maximally. Finally the splittings which generate solar and atmospheric oscillations arise due to breaking of the original symmetry (for example due to additional loop suppression) [13, 14] or due to R-parity breaking [19]. These models lead to a $(2+2)$ scheme.

One important feature of $(3+1)$ mass spectra is that they include the three-active neu- 
trino scenario as limiting case. In this case solar and atmospheric neutrino oscillations are explained by active neutrino oscillations, with mass-squared differences $\Delta m_{\mathrm{SOL}}^{2}$ and $\Delta m_{\mathrm{ATM}}^{2}$, and the fourth neutrino state gets completely decoupled. We will refer to this scenario as $(3+0)$. The $(3+1)$ scheme can be considered as a perturbation of the $(3+0)$ case: a small mixture of $\nu_{e}$ and $\nu_{\mu}$ with the separated mass state can account for the oscillations observed by LSND. In contrast, the $(2+2)$ spectrum is intrinsically different from the three-active neutrino case. A very important prediction of this mass spectrum is that there has to be a significant contribution of the sterile neutrino either in solar or in atmospheric neutrino oscillations or in both. More precisely, in the $(2+2)$ case the fractions of sterile neutrino participating in solar and in atmospheric oscillations have to add up to one [22].

Based on semi-quantitative arguments it has been realized for some time [23, 24, 25, 26] that it is difficult to explain the LSND result in the framework of $(3+1)$ schemes because of strong bounds from negative neutrino oscillation searches in short-baseline (SBL) experiments, and therefore the $(2+2)$ scheme was considered as the preferred one. Recent experimental developments lead to a renaissance of the $(3+1)$ mass schemes [21, 22, 27]. First, a new LSND analysis (see last reference of [10]) resulted in a shift of the region allowed by LSND to slightly smaller values of $\Delta m_{\mathrm{LSND}}^{2}$, which makes the $(3+1)$ schemes somewhat less disfavored. However, in Refs. [28, 29] it was shown within a well defined statistical analysis that a bound implied by SBL experiments is in disagreement even with the new LSND allowed region at the $95 \%$ C.L. in (3+1) schemes. Second, the high statistics data from Super-Kamiokande started to exclude two-neutrino oscillations into a sterile neutrino for both solar as well as atmospheric neutrinos [30], which constitutes a problem for $(2+2)$ mass schemes. Concerning the solar data, the trend to disfavor oscillations into a sterile neutrino recently became supported by the beautiful result of the SNO experiment [5, 31]. However, a unified analysis of solar and atmospheric neutrino data performed in Refs. [32, 33] showed that the goodness of fit of the $(2+2)$ mass scheme is still acceptable.

In this work we perform for the first time a global analysis of all the relevant neutrino oscillation data in a four-neutrino framework. We will use the fit of the global solar neutrino data presented in Ref. [33], which includes Super-Kamiokande [1], Homestake [2], SAGE [3], GALLEX and GNO [4] and SNO [5]. Further we include data from the atmospheric neutrino experiments Super-Kamiokande [7] and MACRO [8], data from the SBL $\stackrel{(-)}{\nu}_{\mu} \rightarrow \stackrel{(-)}{\nu_{e}}$ appearance experiments LSND [11], KARMEN [34] and NOMAD [35], the reactor $\bar{\nu}_{e}$ disappearance experiments Bugey [36] and CHOOZ [37] and the $\stackrel{(-)}{\nu}$ disappearance experiment CDHS [38]. We will perform a fit to these data for $(3+1)$ and $(2+2)$ mass spectra in a unified formalism, which allows to compare directly the quality of the fit for these rather different mass schemes.

The plan of the paper is as follows. In Sec. II we define our notation. In Secs. [II], IV and $\square$ we consider the mixing parameters relevant in the different classes of experiments (SBL, solar and atmospheric, respectively), discuss constraints on these parameters and describe the experimental data used in the analysis. In Sec. $\nabla$ we give a thorough discussion of the 

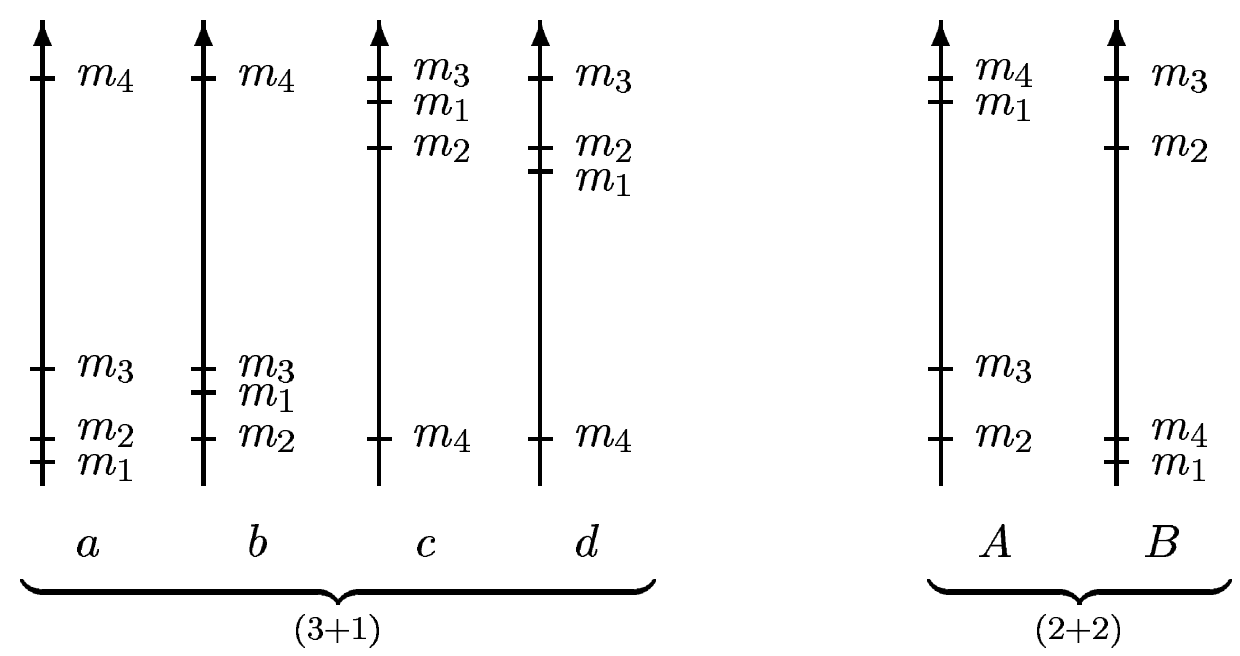

Figure 1: The six types of four-neutrino mass spectra. The different distances between the masses on the vertical axes symbolize the different scales of mass-squared differences required to explain solar, atmospheric and LSND data with neutrino oscillations.

parametrization of four-neutrino mass schemes. The parameterization we introduce is based on physically relevant quantities and is convenient for the combined analysis. In Sec. VII we compare the $(3+1)$ and $(2+2)$ mass schemes when data from solar and atmospheric neutrino experiments are combined, whereas in Sec. VIII we consider the combination of atmospheric and SBL experiments. In Sec. [X] we present the main result of this work: a fit of the global set of neutrino oscillation data (solar, atmospheric and SBL) in the framework of four-neutrino mass spectra. We also comment on a Standard Model fit, i.e. a fit for the $(3+0)$ case. In Secs. VII IX we focus mainly on the relative comparison of $(3+1)$ and $(2+2)$ mass schemes; we will make some comments on the absolute quality of the fit in Sec. $\mathrm{X}$. Finally, we present our conclusions in Sec. XI.

For readers interested mainly in the results of our work we suggest to skip Secs. III VI. After having a look at Fig. 4, where the parameter structure of the four-neutrino fit is illustrated, we recommend to proceed directly to Sec. VII.

\section{FOUR-NEUTRINO OSCILLATION PARAMETERS}

To obtain a four-neutrino scenario from a gauge model of the weak interaction one needs to extend the lepton sector by a number $m$ of $S U(2) \otimes U(1)$ singlet leptons [39]. In such scheme the charged current leptonic weak interaction is specified as by a rectangular $3 \times(3+m)$ lepton mixing matrix $K=\Omega U$ which comes from diagonalizing separately the $3 \times 3$ charged lepton mass matrix (via $\Omega)$ as well as the, in general $(3+m) \times(3+m)$ Majorana, neutrino mass matrix (via $U$ ). Moreover the weak neutral current couplings of mass-eigenstate neutrinos is characterized by a non-trivial $(3+m) \times(3+m)$ coupling matrix $P=K^{\dagger} K$ [39] whose effects will not be relevant for us as neutrinos are both produced and detected through charged 
current interactions. If these extra singlets are all super-heavy (one example is the standard seesaw scheme, where $m=3$ ), they decouple, leaving to a nearly unitary $3 \times 3$ lepton mixing matrix $K$ while the projective $(3+m) \times(3+m)$ matrix $P$ becomes approximately the $3 \times 3$ unit matrix (approximate GIM mechanism).

From here on-wards we assume that, due to some symmetry or another reason [13, 14, 15, 17, 18 one of the $S U(2) \otimes U(1)$ singlets remains light enough so that it can take part in the oscillation phenomenology and thereby account for the LSND data. The minimum possibility is to have just one such light singlet, $m=1$, called sterile neutrino.

In general the physics of four-neutrino oscillations involves 3 mass-squared differences and the elements of the mixing matrix $K$. The latter have been characterized in a modelindependent way in 39] where an explicit parametrization was given which is, up to factor ordering, the standard one. In full generality $K$ contains 6 mixing angles and 3 physical phases which could lead to $\mathrm{CP}$ violation in the oscillation phenomena 40. For convenience this $3 \times 4$ matrix $K$ connecting the 4 neutrino mass fields $\nu_{i}$ and the 3 flavor fields $\nu_{\alpha}$ can be completed with an extra line (relating the sterile neutrino $\nu_{s}$ to the mass eigenstates) so to obtain a $4 \times 4$ unitary matrix. In a basis where the charged lepton mass matrix is diagonal this leads to the matrix $U$ diagonalizing the neutrino mass matrix:

$$
\nu_{\alpha}=\sum_{i=1}^{4} U_{\alpha i} \nu_{i} \quad(\alpha=e, \mu, \tau, s) .
$$

Because of the strong hierarchy of the mass-squared differences required by the experimental data the CP-violating effects are expected to be small in the experiments we consider. However, CP violation can be important in four-neutrino schemes for future long-baseline experiments, such as neutrino factories [41]. Thus, neglecting the complex phases we are left altogether with nine parameters relevant for the description of $\mathrm{CP}$ conserving neutrino oscillations in a four-neutrino scheme: 6 mixing angles contained in $U$ and 3 mass-squared differences. In the following sections we will present a choice for these parameters, which is convenient for the combined analysis of the different experiments and which is motivated by their physical interpretation.

We label the neutrino masses as indicated in Fig. 1 and define for all schemes ${ }^{1}$

$$
\Delta m_{\mathrm{LSND}}^{2}=m_{4}^{2}-m_{2}^{2} \quad \text { and } \quad \Delta m_{\mathrm{ATM}}^{2}=m_{3}^{2}-m_{2}^{2}>0 \text {. }
$$

All experiments we consider are insensitive to the sign of $\Delta m_{\mathrm{LSND}}^{2}$. This implies that the $(3+1)_{a}$ scheme is equivalent to $(3+1)_{d}$, while $(3+1)_{b}$ is equivalent to $(3+1)_{c}$ and $(2+2)_{A}$ is equivalent to $(2+2)_{B} \cdot{ }^{2}$ Hence, without loss of generality we can restrict ourselves to the

\footnotetext{
${ }^{1}$ Note that our labeling is different from the one in previous publications [25, 26, 29, 32]. However, this way of labeling neutrino masses is particularly convenient as it enables a combined treatment of all the schemes in the same footing.

${ }^{2}$ These degeneracies can be lifted by considering the effects in tritium $\beta$-decay experiments [29, 42] or neutrino-less double $\beta$-decay experiments 43 .
} 
discussion of the schemes $(3+1)_{a},(3+1)_{b}$ and $(2+2)_{A}$, and we always have $\Delta m_{\mathrm{LSND}}^{2}>0$. The structure of the neutrino mass eigenstates $\nu_{2}, \nu_{3}$ and $\nu_{4}$ is common for all these schemes. Only the "solar mass state" $\nu_{1}$ is inserted in different places. Let us define the index $\odot$ for the different schemes as

$$
(3+1)_{a}: \odot \equiv 2, \quad(3+1)_{b}: \odot \equiv 3, \quad(2+2): \odot \equiv 4 .
$$

Then the solar mass-splitting can be written for all schemes as

$$
\Delta m_{\mathrm{SOL}}^{2}=m_{\odot}^{2}-m_{1}^{2}>0 .
$$

One advantage of the labeling introduced above is that we can use the parameter $\Delta m_{41}^{2} \equiv$ $m_{4}^{2}-m_{1}^{2}$ to relate the different schemes in a continuous way. The values of $\Delta m_{41}^{2}$ which correspond to the three schemes are given by

$$
\begin{aligned}
(3+1)_{a}: & \Delta m_{41}^{2}=\Delta m_{\mathrm{LSND}}^{2}+\Delta m_{\mathrm{SOL}}^{2} \\
(3+1)_{b}: & \Delta m_{41}^{2}=\Delta m_{\mathrm{LSND}}^{2}+\Delta m_{\mathrm{SOL}}^{2}-\Delta m_{\mathrm{ATM}}^{2}, \\
(2+2)_{A}: & \Delta m_{41}^{2}=\Delta m_{\mathrm{SOL}}^{2} .
\end{aligned}
$$

It will be useful to factorize the mixing matrix $U$ into two matrices: $U=O^{(2)} O^{(1)}$. Neglecting the complex phases in $U$ we write the matrices $O^{(i)}$ as a product of rotation matrices $R_{i j}$ in the $(i, j)$ subspace with the angle $\theta_{i j}$. We define

$$
\begin{aligned}
& O^{(1)}=R_{14} R_{13} R_{12}=\left(\begin{array}{cccc}
c_{14} c_{13} c_{12} & c_{14} c_{13} s_{12} & c_{14} s_{13} & s_{14} \\
-s_{12} & c_{12} & 0 & 0 \\
-s_{13} c_{12} & -s_{13} s_{12} & c_{13} & 0 \\
-s_{14} c_{13} c_{12} & -s_{14} c_{13} s_{12} & -s_{14} s_{13} & c_{14}
\end{array}\right), \\
& O^{(2)}=R_{34} R_{24} R_{23}=\left(\begin{array}{cccc}
1 & 0 & 0 & 0 \\
0 & c_{24} c_{23} & c_{24} s_{23} & s_{24} \\
0 & -s_{34} s_{24} c_{23}-c_{34} s_{23} & -s_{34} s_{24} s_{23}+c_{34} c_{23} & s_{34} c_{24} \\
0 & -c_{34} s_{24} c_{23}+s_{34} s_{23} & -c_{34} s_{24} s_{23}-s_{34} c_{23} & c_{34} c_{24}
\end{array}\right)
\end{aligned}
$$

and order the flavor eigenstates in such a way that if all angles are zero we have the correspondence $\left(\nu_{e}, \nu_{\mu}, \nu_{\tau}, \nu_{s}\right)=\left(\nu_{1}, \nu_{2}, \nu_{3}, \nu_{4}\right)$. In the following sections we will consider the mixing parameters relevant in the three different classes of experiments (SBL, solar and atmospheric) in more detail.

\section{SBL EXPERIMENTS}

\section{A. SBL parameters}

In SBL experiments it is a good approximation to set the solar and atmospheric masssplittings to zero. Obviously, under this assumption the two schemes $(3+1)_{a}$ and $(3+1)_{b}$ 
become equivalent. Let us define the parameters $d_{\alpha}(\alpha=e, \mu, \tau, s)$ and $A_{\mu ; e}$ for the two schemes as

$$
\begin{array}{lll}
(3+1): & d_{\alpha}=\left|U_{\alpha 4}\right|^{2}, & A_{\mu ; e}=4\left|U_{e 4}\right|^{2}\left|U_{\mu 4}\right|^{2}, \\
(2+2): & d_{\alpha}=\left|U_{\alpha 1}\right|^{2}+\left|U_{\alpha 4}\right|^{2}, & A_{\mu ; e}=4\left|U_{e 1} U_{\mu 1}^{*}+U_{e 4} U_{\mu 4}^{*}\right|^{2} .
\end{array}
$$

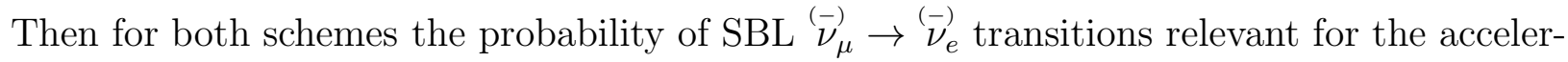
ator experiments LSND, KARMEN and NOMAD is given by

$$
P_{\nu_{\mu} \rightarrow \nu_{e}}=P_{\bar{\nu}_{\mu} \rightarrow \bar{\nu}_{e}}=A_{\mu ; e} \sin ^{2} \frac{\Delta m_{\mathrm{LSND}}^{2} L}{4 E},
$$

and the survival probabilities relevant in the SBL disappearance experiments Bugey and CDHS are given by

$$
P_{\nu_{\alpha} \rightarrow \nu_{\alpha}}=P_{\bar{\nu}_{\alpha} \rightarrow \bar{\nu}_{\alpha}}=1-4 d_{\alpha}\left(1-d_{\alpha}\right) \sin ^{2} \frac{\Delta m_{\mathrm{LSND}}^{2} L}{4 E}
$$

where $\alpha=e$ refers to the Bugey and $\alpha=\mu$ to the CDHS experiment. Here $L$ is the distance between source and detector and $E$ is the neutrino energy. It is straightforward to see that in the $(3+1)$ scheme the relation

$$
(3+1): \quad A_{\mu ; e}=4 d_{e} d_{\mu}
$$

holds. Hence, there are only two independent SBL mixing parameters in this case. However, in the $(2+2)$ scheme the situation is qualitatively different and there is only the restriction

$$
(2+2): \quad A_{\mu ; e} \leq 4 \min \left[d_{e} d_{\mu},\left(1-d_{e}\right)\left(1-d_{\mu}\right)\right]
$$

which follows from unitarity of $U$, and therefore there remain three independent mixing parameters for SBL experiments in the $(2+2)$ scheme.

Note that the probabilities Eqs. (9) and (10) have the same form as in the two-neutrino case [13, 26]. The amplitude $A_{\mu ; e}$ can therefore be identified with the LSND mixing angle:

$$
A_{\mu ; e} \equiv \sin ^{2} 2 \theta_{\mathrm{LSND}},
$$

and for the disappearance parameters the identification $4 d_{e}\left(1-d_{e}\right) \leftrightarrow \sin ^{2} 2 \theta_{\text {Bugey }}$ (and similar for CDHS) can be made.

\section{B. Constraints form Bugey and $\mathrm{CHOOZ}$}

Let us consider the constraints from reactor $\bar{\nu}_{e}$ disappearance experiments Bugey and $\mathrm{CHOOZ}^{3}$ To this purpose we introduce the parameter

$$
\eta_{e} \equiv\left|U_{e 1}\right|^{2}+\left|U_{e \odot}\right|^{2}
$$

\footnotetext{
3 Note that the Palo Verde reactor experiment 44 obtains a bound comparable to CHOOZ. As the exact value of this bound has very little impact on our analysis we include for simplicity only the result of CHOOZ.
} 
which describes the fraction of the electron neutrino in the "solar sector" and is related to $d_{e}$ by

$$
\begin{array}{ll}
(3+1): & \eta_{e}+d_{e} \leq 1, \\
(2+2): & \eta_{e}=d_{e} .
\end{array}
$$

The requirement that the electron neutrino must participate in oscillations with $\Delta m_{\mathrm{SOL}}^{2}$ in order to explain the solar neutrino anomaly leads to $\eta_{e} \sim 1$. The result of the Bugey experiment [36] constrains the combination $4 d_{e}\left(1-d_{e}\right)$ to be very small. Taking into account Eq. (15) and $\eta_{e} \sim 1$ one obtains 26, 28]

$$
\left.\begin{array}{rr}
(3+1): & d_{e} \\
(2+2): & 1-d_{e}
\end{array}\right\} \lesssim 2 \times 10^{-2} \text { at } 90 \% \text { C.L. }
$$

in the relevant range of $\Delta m_{\mathrm{LSND}}^{2}$.

The disappearance probability in the CHOOZ [37] experiment ${ }^{4}$ can be written as

$$
P_{\mathrm{CHOоz}}=1-2 d_{e}\left(1-d_{e}\right)-A_{\mathrm{CHOоz}} \sin ^{2} \frac{\Delta m_{\mathrm{ATM}}^{2} L}{4 E},
$$

with

$$
\begin{array}{ll}
(3+1): & A_{\mathrm{CHOоz}}=4 \eta_{e}\left(1-d_{e}-\eta_{e}\right), \\
(2+2): & A_{\mathrm{CHOоz}}=4\left|U_{e 2}\right|^{2}\left|U_{e 3}\right|^{2} .
\end{array}
$$

We use the result of this experiment in two ways. First, we constrain the SBL parameter $d_{e}$ similar to Bugey as described in Ref. [28]. Second, also the parameter $A_{\mathrm{CHooz}}$ is constrained to small values. Comparing Eqs. (16) and (18) and noting that for the $(2+2)$ schemes $\left(1-d_{e}\right)=\left|U_{e 2}\right|^{2}+\left|U_{e 3}\right|^{2}$ one can see that $A_{\mathrm{CHoOz}}$ is very small in this case because of the bound on $\left(1-d_{e}\right)$ implied by Bugey. However, for the $(3+1)$ schemes the additional information of $\mathrm{CHOOZ}$ is important. Taking again into account that the electron neutrino must have significant mixing with $\nu_{1}$ and $\nu_{\odot}$ to obtain solar neutrino oscillations $\left(\eta_{e} \sim 1\right)$, we obtain the bound

$$
(3+1): \quad 1-d_{e}-\eta_{e} \lesssim 4 \times 10^{-2} \quad \text { at } \quad 90 \% \text { C.L. }
$$

for the values of $\Delta m_{\mathrm{ATM}}^{2}$ preferred by atmospheric neutrino experiments.

To summarize, if oscillations of $\nu_{e}$ with $\Delta m_{\mathrm{SOL}}^{2}$ are required, bounds from reactor experiments imply in both types of mass schemes that $\eta_{e}$ has to be close to 1: for $(2+2)$ Eqs. (15) and (16) imply that $\left(1-\eta_{e}\right)$ is bounded by Bugey, whereas for $(3+1)$ we obtain a somewhat weaker bound resulting from a combination of the bounds from Bugey Eq. (16) and CHOOZ Eq. (19):

$$
\begin{array}{ll}
(3+1): & 1-\eta_{e} \lesssim 6 \times 10^{-2} \\
(2+2): & 1-\eta_{e} \lesssim 2 \times 10^{-2}
\end{array}
$$

\footnotetext{
${ }^{4}$ Due to its value of $(E / L)$ CHOOZ is sensitive to oscillations with $\Delta m_{\mathrm{ATM}}^{2}$ rather than with $\Delta m_{\mathrm{LSND}}^{2}$, and therefore it is considered as a long-baseline experiment. However, it turns out to be convenient to treat it together with the SBL experiments in the analysis.
} 
These bounds can also be translated into bounds on the mixing angles contained in the $O^{(1)}$ factor of the leptonic mixing matrix. One of three angles in the matrix $O^{(1)}$ is the solar angle $\theta_{1 \odot}$, which has to be large 445] in order to account for the results of solar neutrino

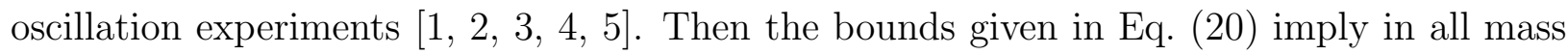
schemes that the other two angles have to be small.

\section{Data used from SBL experiments}

In this section we describe the experimental data from SBL experiments which we are using for our statistical analysis. We divide the $\chi^{2}$-function describing the SBL experiments into two parts:

$$
\begin{aligned}
& \chi_{\mathrm{SBL}}^{2}\left(\Delta m_{\mathrm{LSND}}^{2}, \theta_{\mathrm{LSND}}, d_{e}, d_{\mu}\right)= \\
& \quad \chi_{\mathrm{NEV}}^{2}\left(\Delta m_{\mathrm{LSND}}^{2}, \theta_{\mathrm{LSND}}, d_{e}, d_{\mu}\right)+\Delta \chi_{\mathrm{LSND}}^{2}\left(\Delta m_{\mathrm{LSND}}^{2}, \theta_{\mathrm{LSND}}\right) .
\end{aligned}
$$

Here $\chi_{\mathrm{NEV}}^{2}$ contains the information from the experiments Bugey, CDHS, KARMEN, NO$\mathrm{MAD}$ and $\mathrm{CHOOZ}$, which find no evidence (NEV) for neutrino oscillations, while $\chi_{\mathrm{LSND}}^{2}$ includes the information of LSND, which is the only SBL experiment reporting an evidence for oscillations. For what concerns the parameter dependence shown in Eq. (21) one has to keep in mind that in the $(3+1)$ scheme $\theta_{\mathrm{LSND}}$ is related to $d_{e}$ and $d_{\mu}$ via Eqs. (11) and (13), but in the context of $(2+2)$ schemes all these three parameters are independent.

The Bugey experiment [36] searches for $\bar{\nu}_{e}$ disappearance at the distances $15 \mathrm{~m}, 40 \mathrm{~m}$ and $95 \mathrm{~m}$ away from a nuclear reactor. As input data for our analysis we use Fig. 17 of Ref. [36], where the ratios of the observed events to the number of expected events in case of no oscillations are shown in 25 bins in positron energy for the positions $15 \mathrm{~m}$ and $40 \mathrm{~m}$, and 10 bins for the position $95 \mathrm{~m}$. The CDHS experiment [38] searches for $\nu_{\mu}$ disappearance by comparing the number of events in the so-called back and front detectors at the distances $L_{\text {back }}=885 \mathrm{~m}$ and $L_{\text {front }}=130 \mathrm{~m}$, respectively, from the neutrino source. The data is given in Tab. 1 of Ref. [38] as ratios of these event numbers in 15 bins of "projected range in iron". The KARMEN experiment [34] looks for $\bar{\nu}_{e}$ appearance in a $\bar{\nu}_{\mu}$ beam. We use the number of positron events in 9 bins of positron energy as given in Fig. 2(b) of the second reference in [34. Our re-analysis of the experiments Bugey, CDHS and KARMEN is described in detail in Ref. [28]. To include the results on the $\nu_{\mu} \rightarrow \nu_{e}$ appearance channel obtained by the NOMAD experiment [35] we perform an analysis similar to the one of KARMEN. We use the 14 data points of the energy spectrum of $\nu_{e}$ charged current events given in Fig. 2 of the first reference in [35].

We include the result of the CHOOZ experiment [37] by means of the $\chi^{2}$-function

$$
\chi_{\text {CHоOz }}^{2}=\frac{\left(\left\langle P_{\text {CHOоZ }}\right\rangle-P_{\exp }\right)^{2}}{\sigma_{\text {stat }}^{2}+\sigma_{\text {syst }}^{2}},
$$

where $P_{\exp }=1.01, \sigma_{\text {stat }}=2.8 \%, \sigma_{\text {syst }}=2.7 \%$ [37] and $P_{\text {Сноо }}$ is given in Eq. (17). In the $(2+2)$ case we adopt the approximation $A_{\mathrm{CHоOz}}=0$ (see Sec. [IIB) and hence $\chi_{\text {сHооz }}^{2}$ 
depends only on the parameter $d_{e}$. For the $(3+1)$ case $\chi_{\text {CHоо }}^{2}$ depends on the two independent parameters $d_{e}$ and $\eta_{e}$. Apart from the requirement $\eta_{e} \sim 1$ we are not interested in the exact value of this parameter and we will always minimize with respect to it. In our approximation the $\mathrm{CHOOZ}$ experiment is the only one sensitive to the small value $\left(1-\eta_{e}\right)$ (see following sections), and therefore the minimization with respect to $\eta_{e}$ is trivial and yields $A_{\mathrm{CHOOz}}=0$. Again we are using only the information on $d_{e}$ from CHOOZ in Eq. (21), which is independent of $\Delta m_{\mathrm{ATM}}^{2}$. Therefore, the dependence on $\eta_{e}$ is not shown in Eq. (21).

The total number of data points for all NEV experiments is

$$
N_{\mathrm{NEV}}=60_{(\text {Bugey })}+15_{(\mathrm{CDHS})}+9_{(\mathrm{KARMEN})}+14_{(\mathrm{NOMAD})}+1_{(\mathrm{CHOOZ})}=99 .
$$

To include the detailed structure of the LSND experiment [10, 11] the LSND collaboration [46] has provided us with a table of the likelihood function obtained in the final analysis of their data [11] as a function of the two-neutrino parameters $\Delta m_{\mathrm{LSND}}^{2}$ and $\sin ^{2} 2 \theta_{\mathrm{LSND}}$. Contours of this likelihood function corresponding to $90 \%$ and 99\% C.L. are shown in Fig. 27 of Ref. [11]. The reason that we can use this likelihood function, which was obtained in a two-neutrino analysis, also in the four-neutrino case is that the relevant four-neutrino probability Eq. (9) has the same form as the two-neutrino probability. We include the LSND likelihood function in our analysis by transforming it into a $\chi^{2}$-function according to [47] $\chi^{2}=$ const $-2 \ln \mathcal{L}$. Because of the event-by-event based likelihood analysis performed by the LSND collaboration we cannot use any information on the absolute value of the $\chi^{2}$ function. Therefore, as indicated already in Eq. (21), we use in our analysis only the $\Delta \chi^{2}$ relative to its minimum:

$$
\Delta \chi_{\mathrm{LSND}}^{2}\left(\Delta m_{\mathrm{LSND}}^{2}, \theta_{\mathrm{LSND}}\right) \equiv 2 \ln \mathcal{L}_{\mathrm{LSND}}^{\max }-2 \ln \mathcal{L}_{\mathrm{LSND}}\left(\Delta m_{\mathrm{LSND}}^{2}, \theta_{\mathrm{LSND}}\right) .
$$

In this way we are able to include the LSND data in an optimal way, as we are using directly the analysis performed by the experimental group.

\section{SOLAR NEUTRINO EXPERIMENTS}

For solar neutrino oscillations it is a good approximation to work in the limit $\Delta m_{\mathrm{LSND}}^{2} \rightarrow \infty$ and $\Delta m_{\mathrm{ATM}}^{2} \rightarrow \infty$, so that oscillations induced by the LSND and atmospheric mass-squared differences are completely averaged out. Moreover, in Refs. [32, 33, 48, 49] solar neutrino oscillations in $(2+2)$ schemes has been studied using the approximation $\eta_{e}=1$, which is justified by the Bugey bound Eq. (16). The results obtained there can be applied also to $(3+1)$ mass schemes, if again $\eta_{e}=1$ is adopted. Note however, that in this case only the somewhat weaker bound shown in Eq. (20) applies. The solar oscillation probabilities obtained in these works are valid up to terms of order $\left(1-d_{e}\right)^{2}$ for $(2+2)$ and $\left[d_{e}^{2},\left(1-\eta_{e}\right)^{2}\right]$ for $(3+1)$. Setting $\eta_{e}=1$ reduces the matrix $O^{(1)}$ in all cases to $R_{1 \odot}$, eliminating the other two mixing angles. 
Under these approximations solar neutrino oscillations do not distinguish between $(3+1)$ and $(2+2)$ schemes, and depend only on the three parameters $\Delta m_{\mathrm{SOL}}^{2}, \theta_{\mathrm{SOL}}$ and $\eta_{s}[4]$. The solar mixing angle $\theta_{\mathrm{SOL}}=\theta_{1 \odot}$ is given by

$$
\tan ^{2} \theta_{\mathrm{SOL}} \equiv \frac{\left|U_{e \odot}\right|^{2}}{\left|U_{e 1}\right|^{2}}
$$

and corresponds to $\theta_{12}$ in the notation of Refs. [32, 33]; it can be taken in the interval $0 \leq \theta_{\mathrm{SOL}} \leq \pi / 2$ without loss of generality. The parameter $\eta_{s}$ is defined by

$$
\eta_{s} \equiv\left|U_{s 1}\right|^{2}+\left|U_{s \odot}\right|^{2}
$$

and corresponds to $c_{23}^{2} c_{24}^{2}$ in the notation of Refs. [32, 33]. This parameter describes the fraction of the sterile neutrino participating in solar neutrino oscillations: for $\eta_{s}=0$ solar electron neutrinos oscillate only into active neutrinos, whereas $\eta_{s}=1$ corresponds to pure $\nu_{e} \rightarrow \nu_{s}$ oscillations. ${ }^{5}$ Thus this mixing-type parameter can be interpreted as a model parameter interpolating between the approximate forms for the leptonic mixing matrix given in Refs. [13] and [14, respectively.

To include the information from solar neutrino experiments in our analysis we make use of the results obtained in the four-neutrino analysis performed in Ref. [33]. The experimental data used in this work is the solar neutrino rate of the chlorine experiment Homestake [2], the weighted average rate of the gallium experiments SAGE [0], GALLEX and GNO [四], as well as the 1258-day Super-Kamiokande data sample [1] in form of the recoil electron energy spectrum for both day and night periods, each of them given in 19 data bins, and the recent result from the charged current event rate at SNO [5]. The total number of data points contained in $\chi_{\mathrm{SOL}}^{2}$ is

$$
N_{\mathrm{SOL}}=3_{(\mathrm{Cl}, \mathrm{Ga}, \mathrm{SNO})}+38_{(\mathrm{SK})}=41 .
$$

Details of the solar neutrino analysis can be found in Refs. 31, 33, 51] and references therein.

To include the results of Ref. [33] in our analysis we use $\chi_{\mathrm{SOL}}^{2}$ as a function of $\eta_{s}$ (minimized with respect to the other two parameters $\Delta m_{\mathrm{SOL}}^{2}$ and $\theta_{\mathrm{SOL}}$ ) shown in Fig. 3 of Ref. [33], which we reproduce in Fig. 2. The $\chi^{2}$ is shown relative to the global minimum, which lies in the large mixing angle (LMA) region and has the value $\left(\chi_{\mathrm{SOL}}^{2}\right)_{\min }=35.3$ for $N_{\mathrm{SOL}}-3=38$ degrees of freedom (d.o.f.). The three lines in the figure are obtained by requiring that the solution of the solar neutrino problem lies in the three regions LMA, low/quasi-vacuum (LOW) and small mixing angle (SMA), respectively. Note that $\chi_{\mathrm{SoL}}^{2}\left(\eta_{s}\right)$ is the same for all mass schemes. We clearly see from this figure that solar neutrino data prefers $\eta_{s}=0$, i.e. pure active oscillations. At 99\% C.L. there is the upper bound from the solar data [33]:

$$
\text { solar data: } \eta_{s} \leq 0.52 \text {. }
$$

${ }^{5}$ The parameter $\eta_{s}$ is similar to the parameters $A$ and $c_{s}$, which have been introduced in Refs. [23] and [50], respectively, to describe the effect of $(2+2)$ mass schemes in Big-Bang nucleosynthesis. 


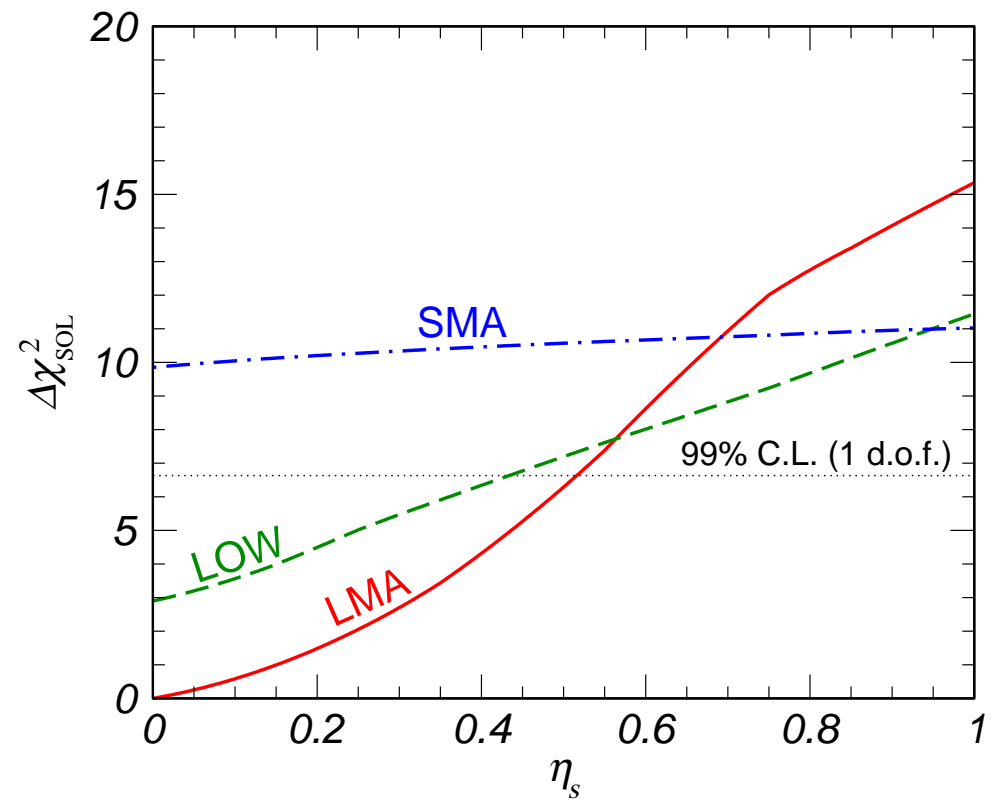

Figure 2: $\Delta \chi_{\mathrm{SOL}}^{2}$ as a function of $\eta_{s}$ for the different solutions to the solar neutrino problem, as presented in Fig. 3 of Ref. [33].

\section{ATMOSPHERIC NEUTRINO EXPERIMENTS}

For the oscillations of atmospheric neutrinos it is a good approximation to set $\Delta m_{\mathrm{SOL}}^{2}$ to zero and to also assume the limit $\Delta m_{\mathrm{LSND}}^{2} \rightarrow \infty$. In Refs. 32, 33, 52 fits of atmospheric neutrino data in a $(2+2)$ framework have been performed by making use of the Bugey constraint Eq. (16) and setting $\eta_{e}=1$. The approximations $\Delta m_{\mathrm{SOL}}^{2}=0$ and $\eta_{e}=1$ imply that the electron neutrino decouples completely from atmospheric neutrino oscillations. In $(3+1)$ spectra the contribution of electron neutrinos to atmospheric oscillations is limited by the somewhat weaker bound shown in Eq. (20); however, in Ref. [51] it was found that a $\nu_{e}$ contamination small enough not to spoil the result of the CHOOZ experiment has only a very small effect on the quality of the fit of atmospheric neutrino data. Therefore, it is justified to adopt the approximation $\eta_{e}=1$ also for $(3+1)$ schemes [29].

Under these assumptions, atmospheric neutrino oscillations do not distinguish between $(3+1)$ and $(2+2)$ schemes, and reduce to an effective three-neutrino problem involving only the flavors $\nu_{\mu}, \nu_{\tau}, \nu_{s}$, the mass eigenstates $\nu_{2}, \nu_{3}, \nu_{4}$ and the mixing matrix $O^{(2)}$ defined in Eq. (77). The $\chi_{\mathrm{ATM}}^{2}$ function depends on the four parameters $\Delta m_{\mathrm{ATM}}^{2}, \theta_{23}, \theta_{34}$ and $\theta_{24}$ [32, 33], and to cover the full physical parameter space one can choose the ranges $0 \leq\left(\theta_{24}, \theta_{34}\right) \leq \pi / 2$ and $-\pi / 2 \leq \theta_{23} \leq \pi / 2 .{ }^{6}$ Therefore, in addition to the two parameters $\Delta m_{\text {Атм }}^{2}$ and $\theta_{\text {Атм }} \equiv$ $\theta_{23}$ corresponding to the two-neutrino parameters, we need two more angles to describe

${ }^{6}$ Note that $\left(\theta_{34}, \theta_{24}, \theta_{23}\right)$ in our notation correspond to $\left(\vartheta_{24}, \vartheta_{23}, \vartheta_{34}\right)$, respectively, in the notation of Refs. [32, 33]. 
atmospheric neutrino oscillations in a four-neutrino framework 48.

To understand the physical meaning of the angles $\theta_{24}$ and $\theta_{34}$ let us consider their relation to the parameters $d_{\mu}$ and $d_{s}$, which we have defined in Eq. (8). Under the approximation $\eta_{e}=1$, we obtain in all the schemes

$$
\begin{aligned}
& d_{\mu}=\left|O_{\mu 4}^{(2)}\right|^{2}=s_{24}^{2}, \\
& d_{s}=\left|O_{s 4}^{(2)}\right|^{2}=c_{24}^{2} c_{34}^{2} .
\end{aligned}
$$

The quantity $\left(1-d_{\mu}\right)\left[\left(1-d_{s}\right)\right]$ corresponds to the fraction of the muon [sterile] neutrino participating in "atmospheric" neutrino oscillations. For $d_{\mu}=s_{24}^{2}=0$ the muon neutrino lies completely in the atmospheric sector, while for the (strongly disfavored) case $d_{\mu}=1$ there are no oscillations of $\nu_{\mu}$ with the scale $\Delta m_{\mathrm{ATM}}^{2}$. Hence, atmospheric data will constrain $d_{\mu}$ to be small. Depending on the value of $\Delta m_{\mathrm{LSND}}^{2}$, the bound on $d_{\mu}$ is strengthened by the $\stackrel{(-)}{\nu}$ SBL disappearance experiment CDHS [25, 29]. Similarly, $d_{s}=c_{24}^{2} c_{34}^{2}=1$ corresponds to pure active atmospheric oscillations, whereas for $d_{s}=0$ the sterile neutrino fully participates in oscillations with $\Delta m_{\mathrm{ATM}}^{2}$. The cases correspond to the approximations used in the early papers [13, 14]: the mixing-type parameter $d_{s}$ can be interpreted as a model parameter interpolating between the approximate forms for the leptonic mixing matrix given in Refs. 13 $\left(d_{s}=0\right)$ and $14\left(d_{s}=1\right)$, respectively.

For the atmospheric data analysis we use the following data from the Super-Kamiokande experiment [7]: $e$-like and $\mu$-like data samples of sub- and multi-GeV, each given as a fivebin zenith-angle distribution, up-going muon data including the stopping ( 5 bins in zenith angle) and through-going (10 angular bins) muon fluxes. Further, we use the recent update of the MACRO [9] up-going muon sample (10 angular bins). We obtain a total number of data points contained in $\chi_{\mathrm{ATM}}^{2}$ of

$$
N_{\text {ATM }}=35_{(\mathrm{SK})}+10_{(\mathrm{MACRO})}=45 .
$$

For further details of the atmospheric neutrino analysis see Refs. [32, 33, 51] and references therein.

In Fig. 3 we show the results of our atmospheric neutrino analysis regarding the angles $\theta_{24}$ and $\theta_{34}$. This figure corresponds to Fig. 6 of Ref. [33], but now using the updated results of MACRO. In the upper panel we show the $90 \%$ and $99 \%$ C.L. allowed regions (2 d.o.f.) for the parameters $d_{s}=c_{24}^{2} c_{34}^{2}$ and $d_{\mu}=s_{24}^{2}$. To obtain these regions we minimize $\chi_{\mathrm{ATM}}^{2}$ with respect to the other two parameters $\theta_{\mathrm{ATM}}$ and $\Delta m_{\mathrm{ATM}}^{2}$. As expected, atmospheric data constrains $d_{\mu}$ to small values, implying a large fraction of $\nu_{\mu}$ participating in atmospheric oscillations. For what concerns the parameter $d_{s}$, values close to 1 are preferred, which means that $\nu_{\mu}$ oscillates mainly to active neutrinos. This can be seen clearly from the lower panel of Fig. 3, where we display $\Delta \chi_{\text {ATM }}^{2}\left(d_{s}\right) \equiv \chi_{\text {АTM }}^{2}\left(d_{s}\right)-\left(\chi_{\text {ATM }}^{2}\right)_{\min }$. Here $\left(\chi_{\text {ATM }}^{2}\right)_{\min }=27.9$ for $N_{\mathrm{ATM}}-4=41$ d.o.f. and $\chi_{\mathrm{ATM}}^{2}$ is minimized with respect to all other parameters. We show the line for the updated MACRO data [9] and compare with the line obtained from the old MACRO data [8], which corresponds to the data used in Ref. [33]. For large values 


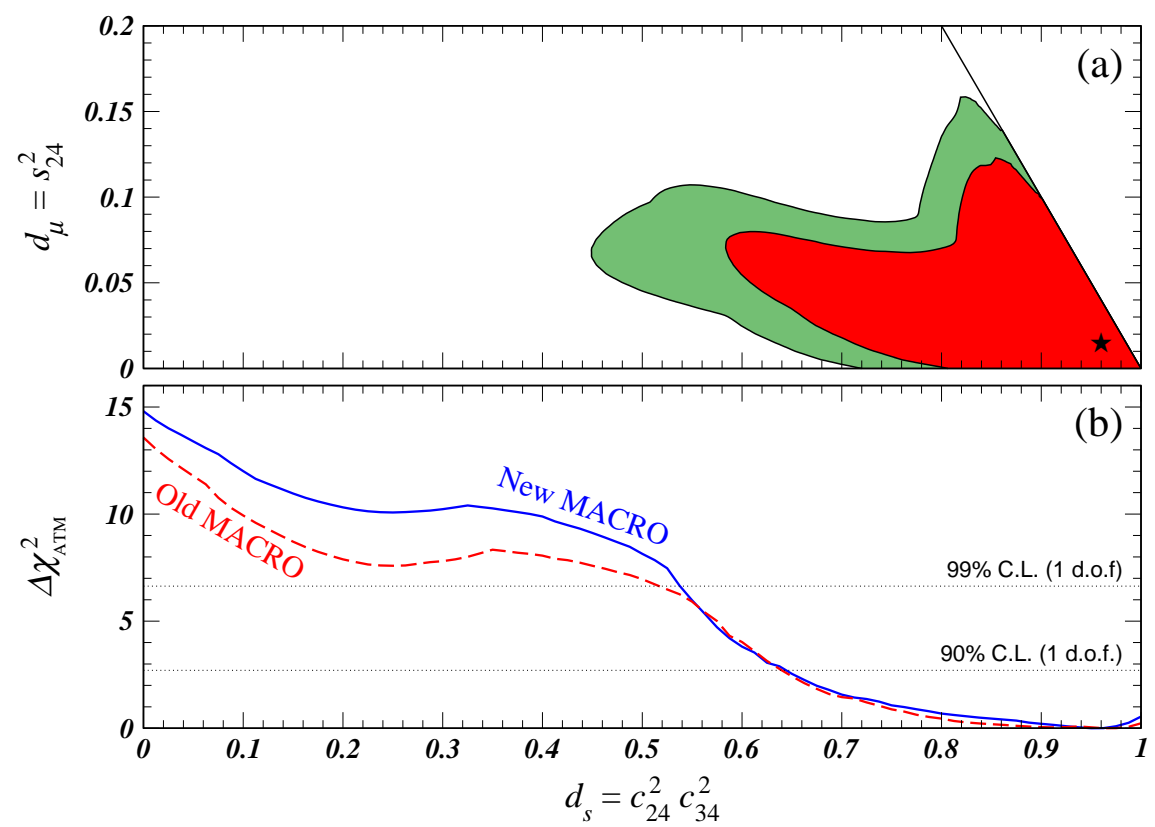

Figure 3: (a) $90 \%$ and $99 \%$ C.L. allowed regions for the parameters $d_{s}=c_{24}^{2} c_{34}^{2}$ (ordinate) and $d_{\mu}=s_{24}^{2}$ (abscissa) from atmospheric neutrino data. The best fit point is marked with a star. (b) $\Delta \chi_{\mathrm{ATM}}^{2}$ as a function of $d_{s}$ using old [8] and new [9] MACRO data. Also shown are the $\Delta \chi^{2}$-values corresponding to $90 \%$ and $99 \%$ C.L. for 1 d.o.f..

\begin{tabular}{l|l}
\hline \hline data set & parameters \\
\hline solar & $\Delta m_{\mathrm{SOL}}^{2}, \theta_{\mathrm{SOL}}, \eta_{s}$ \\
atmospheric & $\Delta m_{\mathrm{ATM}}^{2}, \theta_{\mathrm{ATM}}, \theta_{24}, \theta_{34}$ \\
SBL appearance & $\Delta m_{\mathrm{LSND}}^{2}, \theta_{\mathrm{LSND}}$ \\
SBL disappearance & $\Delta m_{\mathrm{LSND}}^{2}, d_{e}, d_{\mu}$ \\
\hline \hline
\end{tabular}

Table I: Four-neutrino parameters for the different data sets.

of $d_{s}$ the lines are very similar, however for small values the fit gets worse. This means that atmospheric data get stronger in rejecting a sterile component in atmospheric neutrino oscillations.

\section{FOUR-NEUTRINO PARAMETERS IN THE COMBINED ANALYSIS}

In the previous sections we have discussed the parameterization of the four-neutrino problem for the different data sets separately. We summarize our choice of parameters in Tab. 国. Note that for $(3+1)$ schemes $\eta_{e}$ is an additional independent parameter, but we do not list it in Tab. I because in our approximation CHOOZ is the only experiment sensitive to it and we always minimize with respect to it. In $(2+2)$ schemes we have $\eta_{e}=d_{e}$ according to Eq. (15). We have chosen the parameters listed in Tab. [ in such a way that they have 


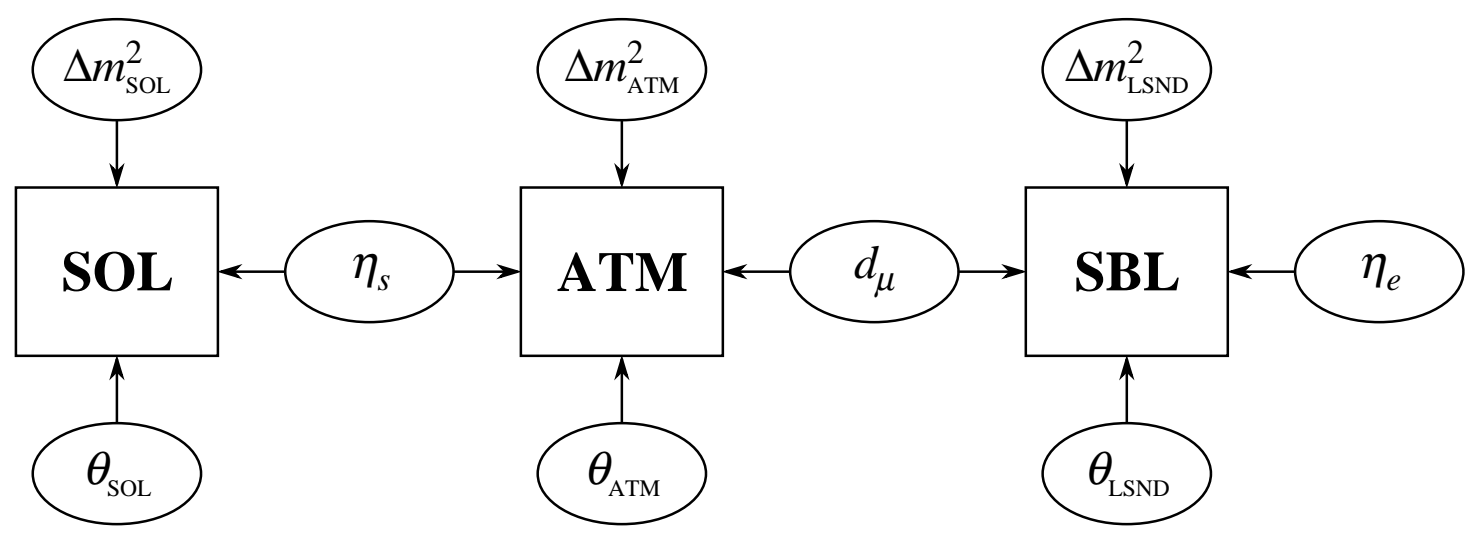

Figure 4: Parameter dependence of the three data sets solar, atmospheric and SBL. Exact definitions of the parameters are given in Secs. $\Pi 1 \mathrm{II}, \mathrm{IV}$ and $\mathrm{V}$.

a well-defined physical meaning in the context of a given data set. Note that this physical interpretation is independent of the mass scheme: for example, regardless of whether we assume $(3+1)$ or $(2+2)$ schemes, $\eta_{s}$ is the fraction of sterile neutrinos in solar oscillations, $\theta_{24}$ describes the fraction of $\nu_{\mu}$ in atmospheric oscillations (see Eq. (29)), and $\sin ^{2} 2 \theta_{\mathrm{LSND}}$ is the SBL $\stackrel{(-)}{\nu}) \rightarrow \stackrel{(-)}{\nu}$ amplitude, and so on. The fact that it is possible to describe the results of any of the given set of experiments in terms of physical quantities independent of the mass scheme implies that none of the considered data sets (solar, atmospheric, SBL appearance or SBL disappearance) can be used on its own to distinguish between different mass spectra. This follows from the approximation $\eta_{e} \approx 1$, which is motivated by the bounds from reactor neutrino experiments, and from the strong hierarchy among the mass-squared differences indicated by the data. This hierarchy implies that for any set of experiments only one mass scale is relevant. In the following we show in detail that the differences between the mass schemes manifest themselves only if two or more data sets are combined, i.e. if the relation among parameters belonging to different data sets is considered.

For the combined analysis we will describe neutrino oscillations by means of the following parameters: beside the three mass-squared differences $\Delta m_{\mathrm{SOL}}^{2}, \Delta m_{\mathrm{ATM}}^{2}$ and $\Delta m_{\mathrm{LSND}}^{2}$ we use the six parameters

$$
\theta_{\mathrm{SOL}}, \theta_{\mathrm{ATM}}, \theta_{\mathrm{LSND}}, \eta_{s}, d_{\mu}, \eta_{e} .
$$

It is easy to check that indeed for all mass schemes these six parameters - defined as in the previous sections - can be used to describe, in a physically more convenient way, the most general CP conserving leptonic mixing matrix [39]. Each of the $\chi^{2}$-functions describing the three data sets (SBL, solar, atmospheric) depends only on a sub-set of these parameters:

$$
\chi_{\mathrm{SOL}}^{2}\left(\Delta m_{\mathrm{SOL}}^{2}, \theta_{\mathrm{SOL}}, \eta_{s}\right), \quad \chi_{\mathrm{ATM}}^{2}\left(\Delta m_{\mathrm{ATM}}^{2}, \theta_{\mathrm{ATM}}, d_{\mu}, \eta_{s}\right), \quad \chi_{\mathrm{SBL}}^{2}\left(\Delta m_{\mathrm{LSND}}^{2}, \theta_{\mathrm{LSND}}, d_{\mu}, \eta_{e}\right) .
$$

We illustrate the parameter dependence of the data sets in Fig. 6 . The three angles $\theta_{\mathrm{SoL}}$, $\theta_{\mathrm{ATM}}, \theta_{\mathrm{LSND}}$ are related directly to the amplitude of the oscillations in the corresponding experiments solar, atmospheric and LSND, while the two quantities $\eta_{s}$ and $d_{\mu}$ account for 
the coupling between different data sets. As indicated, the parameter $\eta_{s}$ is in common to solar and atmospheric neutrino oscillations; if we express it in terms of the atmospheric angles we obtain the different relations, depending on the mass schemes:

$$
\begin{aligned}
(3+1)_{a}: & \eta_{s}=\left|O_{s 2}^{(2)}\right|^{2}=\left(s_{24} c_{34} c_{\mathrm{ATM}}-s_{34} s_{\mathrm{ATM}}\right)^{2} \\
(3+1)_{b}: & \eta_{s}=\left|O_{s 3}^{(2)}\right|^{2}=\left(s_{24} c_{34} s_{\mathrm{ATM}}+s_{34} c_{\mathrm{ATM}}\right)^{2} \\
(2+2): & \eta_{s}=\left|O_{s 4}^{(2)}\right|^{2}=c_{24}^{2} c_{34}^{2} .
\end{aligned}
$$

On the other hand the parameter $d_{\mu}$ is in common to SBL and atmospheric neutrino oscillations; here the coupling is the same in all mass schemes (see Eq. (29)). Another important difference between $(3+1)$ and $(2+2)$ arises due to the combination of SBL appearance and disappearance experiments (see Eqs. (11) and (12)). There is no direct coupling between solar and SBL oscillations, they do not depend on a common parameter. This simple coupling of the data sets is a nice feature of our parameterization (within the adopted approximations) which renders the combined analysis possible, despite the large number of parameters involved. Note that only the SBL experiments involve the additional parameter $\eta_{e}$ explicitly, since - as stated in Secs. $\Pi \nabla$ and $\nabla$ - for what concerns the analysis of the other two data sets it is safe to assume $\eta_{e}=1$. In the $(2+2)$ scheme we have the relation $\eta_{e}=d_{e}$ and this parameter is important for the SBL disappearance amplitude, whereas in $(3+1)$ only the long baseline reactor experiment $\mathrm{CHOOZ}$ is sensitive to $\eta_{e}$ and we always minimize with respect to it.

For the unified analysis of all the mass schemes we will consider $\Delta m_{\mathrm{LSND}}^{2}, \Delta m_{\mathrm{ATM}}^{2}$ and $\Delta m_{41}^{2}$ as the three independent mass-squared differences. The $\chi^{2}$ as a function of $\Delta m_{41}^{2}$ will have three local minima corresponding to the schemes $(3+1)_{a},(3+1)_{b}$ and $(2+2)_{A}$ at the values given in Eq. (5). Beside this parameter indicating the scheme we will display the results of our numerical analysis using the following parameters. For the analysis of solar and atmospheric data in Sec. VII we consider the $\chi^{2}$ as a function of $\eta_{s}$; the results of the analysis of atmospheric and SBL data (Sec. VIII) are given in the $\left(\Delta m_{\mathrm{LSND}}^{2}, \sin ^{2} 2 \theta_{\mathrm{LSND}}\right)$ plane, while for the fully global analysis (Sec. [X] ) we use all three parameters $\left(\Delta m_{\mathrm{LSND}}^{2}, \theta_{\mathrm{LSND}}, \eta_{s}\right)$. Note that in a $\chi^{2}$ analysis the size of the allowed regions depend crucially on the number of parameters considered. Our aim was to identify which parameters describe the most relevant features of the physics in each case.

Before closing this section let us note some subtleties related to our parameterization. As described above, some of the parameters shown in Eq. (31), which we are using for our global fit, obey different relations depending on the mass scheme considered. The question arises of how to treat these different relations among parameters in a common framework for the two mass schemes. Indeed, we are using the parameter $\Delta m_{41}^{2}$ to formally describe a continuous transition between the vastly different mass spectra.

Let us consider a completely arbitrary parameterization of the general four-neutrino problem [39]. We have 3 mass-squared differences and 6 angles, e.g. the angles $\theta_{i j}$ introduced in Eqs. (6) and ([7). Now one can think of a fit to the data in this general parameterization. 


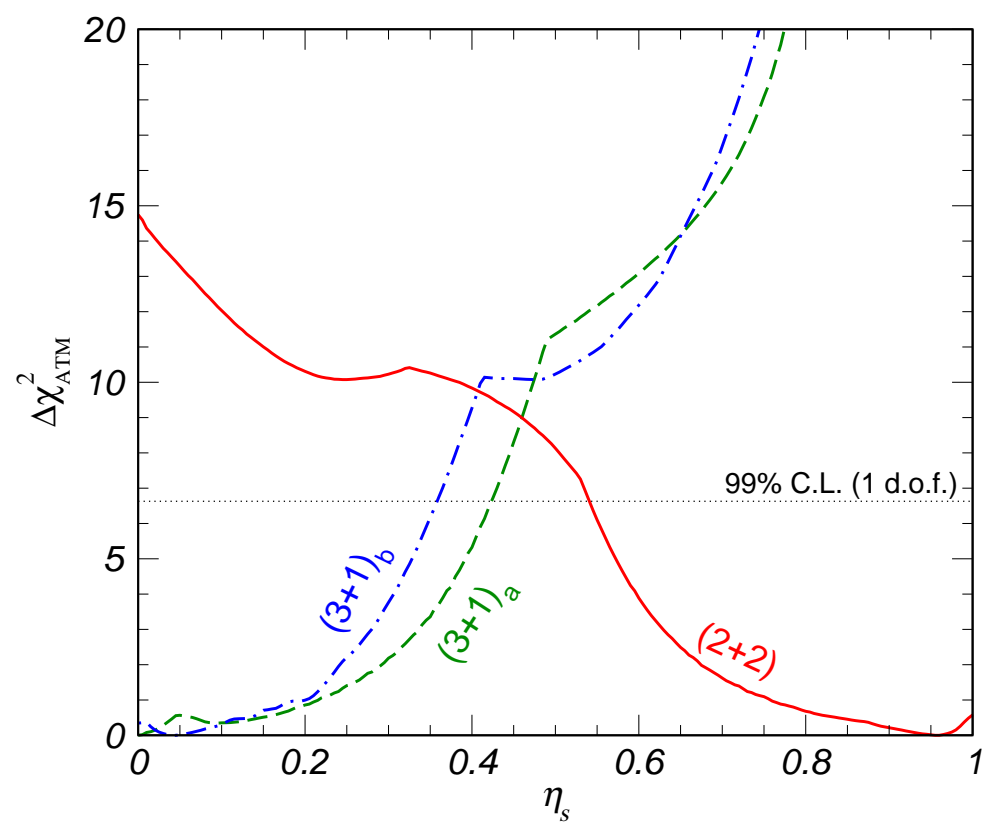

Figure 5: $\Delta \chi_{\mathrm{ATM}}^{2}$ as a function of the fraction of the sterile neutrino in solar oscillations $\eta_{s}$ for all four-neutrino mass schemes, $(3+1)_{a},(3+1)_{b}$ and $(2+2)$.

In practice it is almost impossible to perform this general nine-parameter fit with current computer technology. However, the results of such an analysis would be six well separated regions in the nine-dimensional parameter space, corresponding to the six mass schemes shown in Fig. 1. In these islands our approximations hold and it makes sense to adopt a parameterization motivated by phenomenology. The allowed regions for the parameters $\theta_{i j}$ can be mapped to allowed regions for the parameters shown in Eq. (31), which have a simple physical interpretation. Inside any given island it is clear which relations among the new parameters have to be applied. Obviously this reasoning is only valid under the assumption that various regions are well separated. This assumption can be justified by noting that to move continuously from a $(2+2)$ to a $(3+1)$ scheme one has to break up the hierarchy among the mass-squared differences, and we can expect that at least one data set will give a large $\chi^{2}$ which separates the corresponding allowed regions.

\section{ANALYSIS OF SOLAR AND ATMOSPHERIC NEUTRINO DATA}

In this section we combine solar and atmospheric neutrino data. In Ref. [33] these data have been considered in the $(2+2)$ scheme. Here we discuss some slight changes in this case (due to the updated MACRO data), we extend the analysis also to the case of the $(3+1)$ mass scheme in a way that allows a direct comparison of the fit for these two schemes.

Before combining the two data sets let us consider the impact of atmospheric data alone on the parameter $\eta_{s}$, describing the fraction of sterile neutrinos in solar oscillations. The 
relation of $\eta_{s}$ to the atmospheric parameters is given in Eq. (33) for the three mass schemes $(3+1)_{a},(3+1)_{b}$ and $(2+2)$. In Fig. 5 we show $\Delta \chi_{\mathrm{ATM}}^{2}\left(\eta_{s}\right) \equiv \chi_{\mathrm{ATM}}^{2}\left(\eta_{s}\right)-\left(\chi_{\mathrm{ATM}}^{2}\right)_{\min }$ for the three cases, minimizing with respect to the other parameters $\Delta m_{\mathrm{ATM}}^{2}, \theta_{\mathrm{ATM}}$ and $d_{\mu}$. The line corresponding to the $(2+2)$ scheme is identical to the one shown in the lower panel of Fig. 3 because of the $(2+2)$ relation $\eta_{s}=d_{s}=c_{24}^{2} c_{34}^{2}$ (see Eq. (33)). Atmospheric data prefers large values of $\eta_{s}$, which corresponds to active $\nu_{\mu}$ atmospheric neutrino oscillations. From the figure we can read off the $99 \%$ C.L. bound

$$
\text { atmospheric data: } \eta_{s} \geq 0.54 \text { for }(2+2) \text { schemes }
$$

which is in disagreement with the bound (28) from solar data. On the other hand, concerning $(3+1)$ schemes in Ref. 222 the very interesting fact was noted that atmospheric data give a constraint on the fraction of the sterile neutrino participating in solar oscillations. From Eq. (29) it follows that $\left|O_{s 2}^{(2)}\right|^{2}+\left|O_{s 3}^{(2)}\right|^{2}=1-d_{s}$ is the fraction of sterile neutrinos in atmospheric oscillations which should be small according to the data [30]. Comparing this with Eq. (33) we expect that for $(3+1)$ schemes atmospheric data prefers small values of $\eta_{s}$. Indeed, from Fig. 5 we find the $99 \%$ C.L. bounds

$$
\text { atmospheric data: } \begin{cases}\eta_{s} \leq 0.35 & \text { for }(3+1)_{a} \text { schemes, } \\ \eta_{s} \leq 0.42 & \text { for }(3+1)_{b} \text { schemes }\end{cases}
$$

which are even stronger than the one from solar data Eq. (28).

From Fig. 5 one can see that, although there are quantitative differences between the two schemes $(3+1)_{a}$ and $(3+1)_{b}$, the qualitative behavior is similar. Looking at Eq. (33) it is easy to see that the relation between $\eta_{s}$ and the atmospheric angles in the $(3+1)_{a}$ scheme reduces to the one in the $(3+1)_{b}$ scheme under the transformation $\theta_{\text {Aтм }} \rightarrow \theta_{\text {Атм }}-$ $\pi / 2$. Such a transformation, when applied to the atmospheric oscillation probabilities, is equivalent to changing the sign of $\Delta m_{\mathrm{ATM}}^{2}$, hence we have $\chi_{\mathrm{ATM}}^{2}\left(\Delta m_{\mathrm{ATM}}^{2}, \theta_{\mathrm{ATM}}-\pi / 2, \theta_{24}, \theta_{34}\right)=$ $\chi_{\mathrm{ATM}}^{2}\left(-\Delta m_{\mathrm{ATM}}^{2}, \theta_{\mathrm{ATM}}, \theta_{24}, \theta_{34}\right)$. Therefore, the origin of the difference between the two schemes $(3+1)_{a}$ and $(3+1)_{b}$ can be explained in two different ways. If we require $\Delta m_{\mathrm{ATM}}^{2}>0$, then we end up with two different relations between $\eta_{s}$ and the atmospheric angles $\theta_{\text {ATM }}, \theta_{24}$ and $\theta_{34}$, as we have done so far. Alternatively, if the definition of the atmospheric angles is adjusted so that their relation with $\eta_{s}$ is the same in $(3+1)_{a}$ and $(3+1)_{b}$ schemes, then it is no longer sufficient to restrict to the case $\Delta m_{\mathrm{ATM}}^{2}>0$, and the case $\Delta m_{\mathrm{ATM}}^{2}<0$ should be investigated as well. In the latter approach, it is clear that the difference arise because of the presence of matter effects in atmospheric neutrino oscillations, which are sensitive to the sign of $\Delta m_{\mathrm{ATM}}^{2}$. Since in this work we are mainly interested in the comparison of $(2+2)$ with $(3+1)$ in general, from now on we will always minimize with respect to $(3+1)_{a}$ and $(3+1)_{b}$ by choosing from the two corresponding values of $\Delta m_{41}^{2}$ the one with the lower $\chi^{2}$.

From Eqs. (28), (34) and (35) one expects that combined solar and atmospheric neutrino data will prefer $(3+1)$ mass schemes over $(2+2)$. In order to quantify this statement let us 

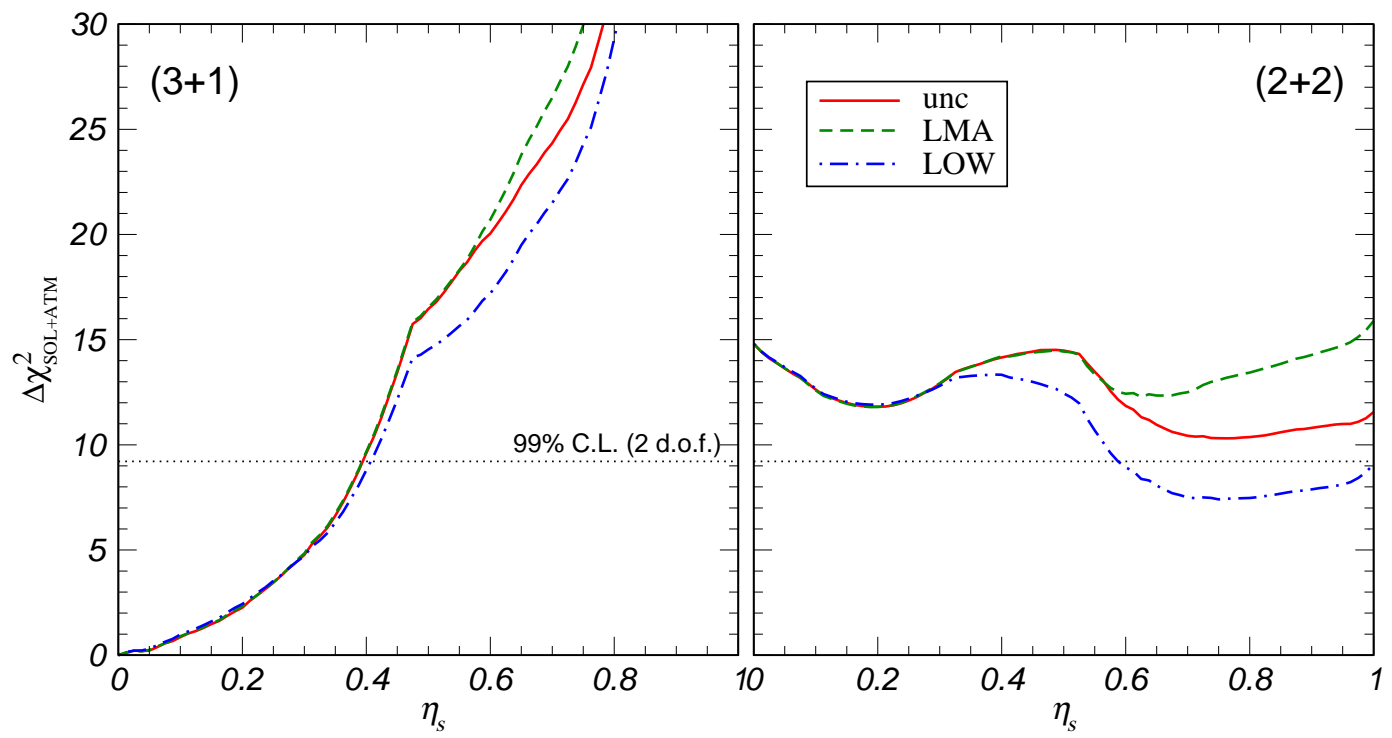

Figure 6: Combined $\chi^{2}$-function for solar and atmospheric neutrino data for $(3+1)$ and $(2+2)$ for different solar neutrino solutions with respect to the global minimum (see text for details).

consider the following $\chi^{2}$-function:

$$
\chi_{\mathrm{SOL}+\mathrm{ATM}}^{2}\left(\eta_{s}, \Delta m_{41}^{2}\right) \equiv \chi_{\mathrm{SOL}}^{2}+\chi_{\mathrm{ATM}}^{2}
$$

where we minimize with respect to the parameters $\Delta m_{\mathrm{SOL}}^{2}, \Delta m_{\mathrm{ATM}}^{2}, \theta_{\mathrm{SOL}}, \theta_{\mathrm{ATM}}$ and $d_{\mu}$. As explained before (see Sec. II) the parameter $\Delta m_{41}^{2}$ relates the different schemes. In Fig. [6 we show the $\Delta \chi^{2}$ projected onto the $\eta_{s}$-axis for the two regions of $\Delta m_{41}^{2}$ corresponding to the schemes $(3+1)$ and $(2+2)$ according to Eq. (5). In both cases, $(3+1)$ and $(2+2)$, we refer to the same minimum, which occurs for the $(3+1)$ scheme with $\eta_{s}=0$. For the dashed (dashed-dotted) line we restrict the solar solution to be LMA (LOW), while for the solid line (labeled "unc" in the figure) we choose the solution which gives the weakest restriction (unconstrained). This corresponds to our current knowledge of the solution to the solar neutrino problem. The reason why the line for LOW sometimes is below the one for the unconstrained case is that it is referred to the minimum for the LOW solution, which is higher than the minimum for LMA (which coincides with the minimum in the unconstrained case) as can be seen from Fig. 2. For the unconstrained case the minimum has the value $\left(\chi_{\mathrm{SOL}+\mathrm{ATM}}^{2}\right)_{\min }=63.2$ for $N_{\mathrm{SOL}}+N_{\mathrm{ATM}}-7=79$ d.o.f.

As expected, solar and atmospheric neutrino data prefer $(3+1)$ because in this case both can be explained by active neutrino oscillations. The dotted line in Fig. of shows the value of $\Delta \chi^{2}=9.21$ corresponding to $99 \%$ C.L. for 2 d.o.f., which are the two parameters $\eta_{s}$ and $\Delta m_{41}^{2}$. Therefore, for the unconstrained and LMA cases $(2+2)$ is disfavored at more than $99 \%$ C.L. with respect to $(3+1)$. If we compare the local minimum with respect to $\eta_{s}$ in $(2+2)$ with the global minimum in $(3+1)$ we find for the unconstrained case

$$
\Delta \chi^{2}=\left(\chi_{\mathrm{SOL}+\mathrm{ATM}}^{2(2+2)}\right)_{\min }-\left(\chi_{\mathrm{SOL}+\mathrm{ATM}}^{2(3+1)}\right)_{\min }=10.3 .
$$




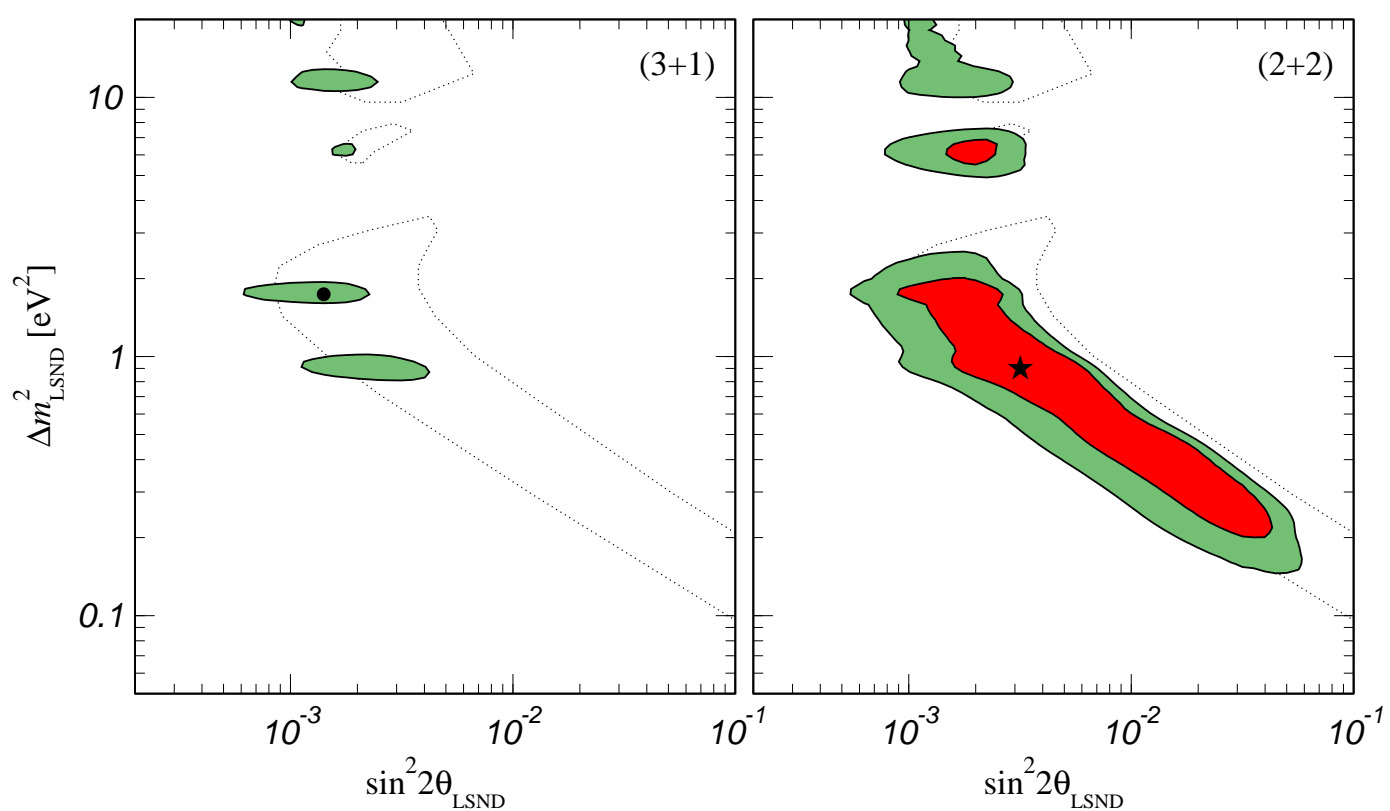

Figure 7: Combination of atmospheric and SBL data. We show projections of the three-dimensional $90 \%$ and $99 \%$ C.L. regions corresponding to $(3+1)$ and $(2+2)$ in the $\left(\Delta m_{\mathrm{LSND}}^{2}, \sin ^{2} 2 \theta_{\mathrm{LSND}}\right)$ plane. The best fit point lies in $(2+2)$ and is marked with a star, the local best fit point in $(3+1)$ is marked with a circle. The doted line is the $99 \%$ C.L. region from LSND data alone [11].

Conversely, the LOW $(2+2)$ solution is still allowed at the 99\% C.L.. The reason for this is, that the LOW solution is not as strong to reject a sterile component in solar oscillations as the LMA solution (see Fig. 2), so that the disagreement with atmospheric data in the context of $(2+2)$ schemes is somewhat weaker. Let us note that for the unconstrained case large values of $\eta_{s} \sim 0.76$ (corresponding to a large component of sterile neutrino in solar oscillations) are slightly preferred over small ones. This means that the inclusion of the updated MACRO results makes atmospheric neutrino data slightly more powerful to reject the sterile neutrino than the unconstrained solar data.

\section{ANALYSIS OF ATMOSPHERIC AND SBL NEUTRINO DATA}

In this section we combine the data sets from atmospheric and SBL neutrino experiments. To this purpose we consider the $\chi^{2}$-function

$$
\chi_{\mathrm{ATM}+\mathrm{SBL}}^{2}\left(\Delta m_{41}^{2}, \Delta m_{\mathrm{LSND}}^{2}, \theta_{\mathrm{LSND}}\right)=\chi_{\mathrm{ATM}}^{2}+\chi_{\mathrm{SBL}}^{2} .
$$

From Eq. (32) we can see that the terms on the right hand side in general depend on the parameters $\left(\Delta m_{\mathrm{LSND}}^{2}, \Delta m_{\mathrm{ATM}}^{2}, \theta_{\mathrm{ATM}}, \theta_{\mathrm{LSND}}, \eta_{s}, d_{\mu}, \eta_{e}\right)$. To obtain the parameter dependence as shown in Eq. (38) we proceed as follows. First, we minimize $\chi_{\text {AтM }}^{2}$ with respect to $\Delta m_{\text {Атм }}^{2}, \theta_{\text {Aтм }}$ and $\eta_{s}$. In a second step we minimize with respect to $d_{e}$ and $d_{\mu}$ by taking into account the relation (11) or (12), depending on the mass scheme considered (i.e. on the value of $\Delta m_{41}^{2}$ ). 
The allowed regions in the parameter space $\left(\Delta m_{41}^{2}, \Delta m_{\mathrm{LSND}}^{2}, \theta_{\mathrm{LSND}}\right)$ are given by $\Delta \chi^{2}=6.3$ (11.3) for 90\% (99\%) C.L. (3 d.o.f.). In the right panel of Fig. 7 we show a projection of the three-dimensional regions corresponding to the $(2+2)$ case, which include the best fit point $\left(\Delta m_{\mathrm{LSND}}^{2}=0.91 \mathrm{eV}^{2}, \sin ^{2} 2 \theta_{\mathrm{LSND}}=3.16 \times 10^{-3}\right)$. One can see that the allowed regions cover a large part of the two-neutrino allowed region by LSND alone, which is shown as the dotted line. The allowed region disappears for values of $\sin ^{2} 2 \theta_{\text {LSND }} \gtrsim 0.06$ because of the constraint from the Bugey experiment. At large values of $\Delta m_{\mathrm{LSND}}^{2}$ the bounds from KARMEN and NOMAD are important. In the left panel we show the projection of the three-dimensional volume corresponding to the $(3+1)$ case with respect to the global minimum, which lies in the $(2+2)$ plane. Only four small islands appear at $99 \%$ C.L.. If we compare the local best fit point for $(3+1)$ at $\left(\Delta m_{\mathrm{LSND}}^{2}=1.74 \mathrm{eV}^{2}, \sin ^{2} 2 \theta_{\mathrm{LSND}}=1.41 \times 10^{-3}\right)$ with the global best fit point we find

$$
\Delta \chi^{2}=\left(\chi_{\mathrm{ATM}+\mathrm{SBL}}^{2(3+1)}\right)_{\min }-\left(\chi_{\mathrm{ATM}+\mathrm{SBL}}^{2(2+2)}\right)_{\min }=6.9 .
$$

The conclusion from Fig. 7 and Eq. (39) is that SBL data combined with atmospheric data clearly prefer $(2+2)$ over the $(3+1)$ spectra. Fig. 7 is a beautiful confirmation of the results of our previous work [29], where we have analyzed a similar data set in the $(3+1)$ framework, but using a very different statistical method. The reason for the $(2+2)$ preference by the SBL data is well known [22, 23, 24, 25, 26, 27] and can be understood from Eqs. (11) and (12). For the $(3+1)$ case both $d_{e}$ and $d_{\mu}$ must be small because of Bugey and CDHS, respectively, which leads to a double suppression of the LSND amplitude $\sin ^{2} 2 \theta_{\text {LSND }}$ according to Eq. (11). In contrast, for the $(2+2)$ case $\left(1-d_{e}\right)$ and $d_{\mu}$ have to be small and Eq. (12) implies only a linear suppression of $\sin ^{2} 2 \theta_{\text {LSND }}$.

\section{GLOBAL ANALYSIS}

The results of the previous section, i.e. that atmospheric+SBL data prefer $(2+2)$ over $(3+1)$, are in direct conflict with the results of Sec. VII, where we have found that solar+atmospheric data prefer $(3+1)$ over $(2+2)$. This shows that there is some tension in the existing data in a four-neutrino framework, and to clarify the situation it is necessary to perform a combined analysis of all the data. To this end we consider the $\chi^{2}$-function

$$
\chi_{\text {global }}^{2}\left(\Delta m_{41}^{2}, \Delta m_{\mathrm{LSND}}^{2}, \theta_{\mathrm{LSND}}, \eta_{s}\right)=\chi_{\mathrm{SOL}}^{2}+\chi_{\mathrm{ATM}}^{2}+\chi_{\mathrm{SBL}}^{2}
$$

We minimize the right-hand side of this equation with respect to all the parameters, except the ones shown on the left-hand side. Allowed regions are given by

$$
\Delta \chi_{\text {global }}^{2}=\chi_{\text {global }}^{2}-\left(\chi_{\text {global }}^{2}\right)_{\min }=7.8(13.3)
$$

for $90 \%$ (99\%) C.L. (4 d.o.f.). From this equation we obtain two four-dimensional volumes in the space of $\left(\Delta m_{41}^{2}, \Delta m_{\mathrm{LSND}}^{2}, \sin ^{2} 2 \theta_{\mathrm{LSND}}, \eta_{s}\right)$ corresponding to $(3+1)$ and $(2+2)$, which we display in Fig. 8 in the following way. In the lower panels we show projections of the 

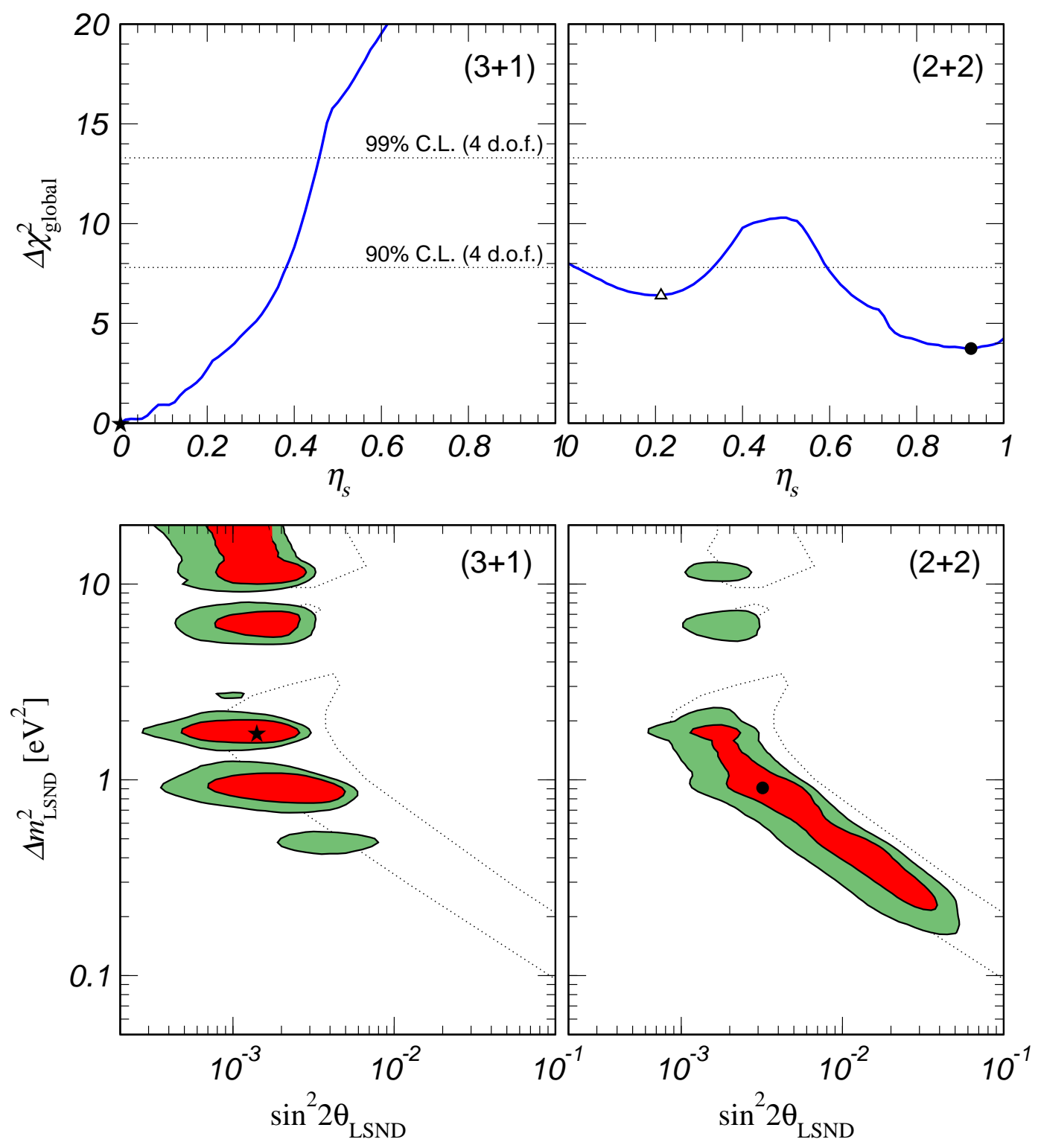

Figure 8: Global combination of current neutrino oscillation data: solar, atmospheric and SBL. We show the $\Delta \chi_{\text {global }}^{2}$ as a function of $\eta_{s}$ (upper panels) and projections of the four-dimensional $90 \%$ and $99 \%$ C.L. regions on the $\left(\Delta m_{\mathrm{LSND}}^{2}, \sin ^{2} 2 \theta_{\mathrm{LSND}}\right)$ plane (lower panels) (see text for details). The global best fit point lies in $(3+1)$ and is marked with a star, the local best fit point in $(2+2)$ is marked with a circle and the local minimum in $(2+2)$ is marked with a triangle. The doted line in the lower panels is the 99\% C.L. region from LSND data alone [11].

four-dimensional volumes onto the $\left(\Delta m_{\mathrm{LSND}}^{2}, \sin ^{2} 2 \theta_{\mathrm{LSND}}\right)$ plane. In the upper panels we show $\Delta \chi_{\text {global }}^{2}$ minimized with respect to all parameters except $\eta_{s}$. The projections of the fourdimensional $90 \%$ and $99 \%$ C.L. volumes onto the $\eta_{s}$-axis are given by the intersections of the solid lines in the upper panels with the corresponding horizontal dotted lines.

Let us discuss the results of the global analysis. We find that the global minimum lies in 


\begin{tabular}{l|ccccc}
\hline \hline & $\Delta m_{\mathrm{LSND}}^{2}\left[\mathrm{eV}^{2}\right]$ & $\sin ^{2} 2 \theta_{\mathrm{LSND}}$ & $\eta_{s}$ & $d_{\mu}$ & $d_{e}$ \\
\hline global best fit (3+1) & 1.74 & $1.41 \times 10^{-3}$ & 0.0 & $1.98 \times 10^{-2}$ & $1.79 \times 10^{-2}$ \\
best fit (2+2) & 0.87 & $3.55 \times 10^{-3}$ & 0.93 & $6.56 \times 10^{-3}$ & 0.99275 \\
local minimum (2+2) & 0.87 & $3.55 \times 10^{-3}$ & 0.21 & $1.32 \times 10^{-2}$ & 0.99275 \\
\hline \hline
\end{tabular}

Table II: Parameter values at the best fit points in $(3+1)$ and $(2+2)$ and at the local minimum in $(2+2)$.

the $(3+1)$ scheme. This minimum is marked as a star in Fig. 8. In $(2+2)$ there are two local minima: the $(2+2)$ best fit point is marked with a circle and corresponds to large values of $\eta_{s}$, whereas the second local minimum (marked with a triangle) occurs for small $\eta_{s}{ }^{7}$ The values of the parameters at these minima are given in Tab. II. However, the difference between the best fit points in $(3+1)$ and $(2+2)$ is not very big:

$$
\Delta \chi^{2}=\left(\chi_{\text {global }}^{2(2+2)}\right)_{\min }-\left(\chi_{\text {global }}^{2(3+1)}\right)_{\min }=3.7
$$

We conclude that the schemes $(3+1)$ and $(2+2)$ give a comparable global fit to the data. This can also be seen from the fact that there are large allowed regions for both mass spectra. The conflicting values given in Eq. (37) (for solar and atmospheric data) and in Eq. (39) (for atmospheric and SBL data) cancel each other to some extent. Solar plus atmospheric data seem to be stronger than SBL data, therefore $(3+1)$ is slightly preferred over $(2+2)$ in the global fit to current neutrino oscillation data.

The shape of the allowed regions in the $\left(\Delta m_{\mathrm{LSND}}^{2}, \sin ^{2} 2 \theta_{\mathrm{LSND}}\right)$ plane for $(2+2)$ (lower right panel of Fig. 8) is simliar to the one expected from a two-neutrino analysis of SBL data alone. In the region $0.18 \mathrm{eV}^{2} \lesssim \Delta m_{\mathrm{LSND}}^{2} \lesssim 8 \mathrm{eV}^{2}$ they follow closely the two-neutrino LSND region. The slight shift to smaller values of $\Delta m_{\mathrm{LSND}}^{2}$ is because of the constraint from KARMEN. Large values of $\sin ^{2} 2 \theta_{\mathrm{LSND}} \gtrsim 0.06$ are excluded by Bugey and for $\Delta m_{\mathrm{LSND}}^{2} \gtrsim 10 \mathrm{eV}^{2}$ constraints from KARMEN and NOMAD are important. ${ }^{8}$ In contrast, in the $(3+1)$ case (lower left panel of Fig. 8) the allowed regions consist of several islands and are very different from the twoneutrino ones. The most prominent islands are at the values $\Delta m_{\mathrm{LSND}}^{2} \sim 0.9,1.7,6 \mathrm{eV}^{2}$ and $\sin ^{2} 2 \theta_{\mathrm{LSND}} \sim 10^{-3}$. These are the values of $\Delta m_{\mathrm{LSND}}^{2}$ where the bounds of all NEV experiments have some marginal overlap with the LSND allowed region [21, 22, 27, 28, 29. However, at $99 \%$ C.L. appears an allowed region at $\Delta m_{\mathrm{LSND}}^{2} \sim 0.5 \mathrm{eV}^{2}$, and a very marginal island at $\Delta m_{\mathrm{LSND}}^{2} \sim 2.5 \mathrm{eV}^{2}$. There is also an allowed region for large values of $\Delta m_{\mathrm{LSND}}^{2} \gtrsim 10 \mathrm{eV}^{2}$. However, in this region there are further constraints from experiments not included in our

\footnotetext{
${ }^{7}$ Note that the two stars in the lower and upper panels actually correspond to the same single point in the four-dimensional space. The same holds for the two circles. We do not show the triangle in the lower right panel, because it would coincide with the circle (see Tab. II).

8 All the relevant SBL bounds are shown e.g. in Fig. 27 of Ref. 11]. A combined analysis of LSND and KARMEN in a two-neutrino framework has been performed in Ref. [53].
} 
analysis, which are BNL E776 [54 $(\stackrel{(-)}{\nu}) \rightarrow \stackrel{(-)}{\nu})_{e}$ appearance $)$ and CCFR 55 $\left.(\stackrel{(-)}{\nu}) \rightarrow \stackrel{(-)}{\nu}\right)_{e}$ appearance and $\stackrel{(-)}{\nu_{\mu}}$ disappearance). Therefore, we do not display values of $\Delta m_{\mathrm{LSND}}^{2}>20 \mathrm{eV}^{2}$.

Discussing the upper panels of Fig. 8 we note that, as already found in Sec. VII, large values of $\eta_{s}$ are preferred for the $(2+2)$ case. Comparing the shape of the $\chi^{2}$ in the global analysis with the one shown in Fig. 6 we observe that the inclusion of SBL data strengthen this trend to some extend. This implies a large component of the sterile neutrino in solar neutrino oscillations and corresponds to the LOW/quasi-vacuum solution of the solar neutrino problem (see Fig. 2). But also the local minimum for smaller $\eta_{s}$ values, which corresponds to the LMA solar solution and implies a large sterile component in atmospheric oscillations, is well inside the $90 \%$ C.L. region. The difference in $\chi^{2}$ between the two local minima in $(2+2)$ is 2.7 . Moreover, the minima in $\chi^{2}$ are not very deep so that all values of $\eta_{s}$ between 0 and 1 are within the 99\% C.L. region. Only values around 0.5 are excluded at $90 \%$ C.L.. The results shown in Fig. 8 were obtained by using unconstrained solar data. We have also performed the analysis by restricting solar data to the LMA and LOW region. The results are very similar to the unconstrained case. For the LMA solution we obtain approximately the solution corresponding to the local minimum in $(2+2)$, which means that $(2+2)$ is sightly more disfavored against $(3+1)$, whereas for the LOW case the difference would become even smaller than shown in Eq. (42).

Recently, solar neutrino data have been analyzed using a new prediction of the ${ }^{8} \mathrm{~B}$ flux [56]. From Tab. 3 of Ref. [56] one can see that LMA becomes relatively better than LOW. This would lead to an up-wards shift of the LOW line in Fig. 2 of approximately 3 units. Consequently solar data becomes stronger in rejecting the sterile neutrino. Regarding the fourneutrino analysis, this would disfavor the $(2+2)$ scheme slightly more against the $(3+1)$ case.

In our framework it is also possible to test the fit of the $(3+0)$ scenario, where the solar and atmospheric neutrino problems are explained by oscillations among three active neutrinos and the explanation of LSND is left out. This would correspond to the Standard Model situation. We obtain this case by considering the $(3+1)$ scheme (this fixes the parameter $\left.\Delta m_{41}^{2}\right)$ and setting the parameters $d_{e}=d_{\mu}=\eta_{s}=0$. Then the sterile neutrino is completely decoupled and we are left with three active neutrinos and the mass splittings $\Delta m_{\mathrm{SOL}}^{2}$ and $\Delta m_{\mathrm{ATM}}^{2}$. We find a difference in $\chi^{2}$ to the best fit point of

$$
\Delta \chi^{2}=\chi_{\text {global }}^{2(3+0)}-\left(\chi_{\text {global }}^{2(3+1)}\right)_{\min }=19.8 .
$$

For 4 d.o.f. $\left(d_{e}, d_{\mu}, \eta_{s}, \Delta m_{41}^{2}\right)$ this corresponds to an exclusion at more than $99.9 \%$ C.L. ${ }^{9}$ We conclude that the data of LSND (using the result of the analysis performed by the LSND collaboration) plays a very significant role and that the global fit in a four-neutrino scenario is much better than in the three-active neutrino case.

\footnotetext{
${ }^{9}$ Regarding the exact value of this C.L. see also the discussion of the $(3+0)$ case in the next section.
} 


\section{GOODNESS OF FIT}

In the previous sections we have restricted ourselves to the relative comparison of the fit in the different mass schemes. Here we discuss the absolute goodness of fit (GOF). A common way of evaluating the GOF is to consider the absolute value of the $\chi^{2}$-function at the best fit point. We are aware of the fact that GOF-values obtained in this way are not very restrictive in a global analysis with many data points like in our case. Therefore, we will also consider in this section the quality of the fit in the four-neutrino schemes $(3+1)$, $(2+2)$ and for the three active neutrino case $(3+0)$ for each of the different data sub-sets separately.

As explained in Sec. III we are not able to use any information on the absolute value of $\chi_{\mathrm{LSND}}^{2}$ from the LSND data. However, let us note that the fit for LSND is expected to be rather good. In Ref. [11] the $\chi^{2}$ for the fit of the $L / E$ distribution to the decay-at-rest events of the LSND data is given for two typical values of $\Delta m_{\mathrm{LSND}}^{2}$ as $\chi^{2}=4.9$ and 5.8 for 8 d.o.f., which corresponds to a very good GOF of $77 \%$ and $67 \%$, respectively. From Fig. 8 one can see that for $(3+1)$ as well as for $(2+2)$ the best fit point lies well inside the $99 \%$ C.L. region of LSND. Therefore, we expect that the contribution of LSND will not worsen the global fit significantly (see also Tab. III).

In the following we evaluate the $\chi^{2}$-functions for all experiments except LSND

$$
\chi_{\text {global-LSND }}^{2} \equiv \chi_{\mathrm{SOL}}^{2}+\chi_{\mathrm{ATM}}^{2}+\chi_{\mathrm{NEV}}^{2}
$$

at the global best fit point in $(3+1)$ and the best fit point in $(2+2)$ :

$$
\begin{array}{ll}
(3+1): & \chi_{\text {global-LSND }}^{2}=150.0 / 176 \text { d.o.f. } \\
(2+2): & \chi_{\text {global-LSND }}^{2}=156.1 / 176 \text { d.o.f.. }
\end{array}
$$

The number of d.o.f. is given by (see Eqs. (23), (27), (30)) $N_{\mathrm{SOL}}+N_{\mathrm{ATM}}+N_{\mathrm{NEV}}=185$

minus 9 fitted parameters. Usually a fit is considered to be good if the value of the $\chi^{2}$ is approximately equal to the number of d.o.f..

The GOF implied by the $\chi^{2}$-values and the corresponding number of d.o.f. given in Eq. (45) would be excellent for both schemes. However, one has to be careful in the interpretation of these numbers. This $\chi^{2}$-test for the GOF is not a very restrictive criterion in a global fit of different experiments with a large number of data points and many parameters. One reason is that in such a case a given parameter is constrained often only by a small sub-set of the data. The rest of the data (which can contain many data points) is fitted perfectly by this parameter (because it is insensitive to it). A discussion of this problem can be found in Ref. [57] or in the context of solar neutrino analysis in Refs. [58.

In order to obtain more insight in the quality of the global fit we will consider the following quantities:

$$
\Delta \chi_{\sigma}^{2}=\chi_{\sigma}^{2}(\alpha)-\left(\chi_{\sigma}^{2}\right)_{\min }
$$




\begin{tabular}{l|c|l|ccrr}
\hline \hline data set & d.o.f. & parameters & $(3+1)$ & $(2+2)_{\text {best }}$ & $(2+2)_{\text {local }}$ & $(3+0)$ \\
\hline \multirow{2}{*}{ solar } & 3 & $\Delta m_{\mathrm{SOL}}^{2}, \theta_{\mathrm{SOL}}, \eta_{s}$ & 0.0 & 10.7 & 1.6 & 0.0 \\
atmospheric & 4 & $\Delta m_{\mathrm{ATM}}^{2}, \theta_{\mathrm{ATM}}, \eta_{s}, d_{\mu}$ & 0.0 & 0.2 & 11.5 & 0.3 \\
LSND & 2 & $\Delta m_{\mathrm{LSND}}^{2}, \theta_{\mathrm{LSND}}$ & 3.0 & 0.7 & 0.7 & 29.0 \\
$\mathrm{NEV}$ & $2 / 3$ & $\theta_{\mathrm{LSND}}, d_{e}, d_{\mu}$ & 8.8 & 3.7 & 4.1 & 2.3 \\
\hline \hline
\end{tabular}

Table III: $\Delta \chi^{2}$ for the different data sets of the best fit points in $(3+1)$ and $(2+2)$, the local minimum in $(2+2)$ and for the $(3+0)$ case (see text for details). Also shown is the number of d.o.f. and the corresponding parameters for each data set.

Here $\chi_{\sigma}^{2}$ is the $\chi^{2}$-function of the data set $\sigma=$ SOL, ATM, LSND, NEV and $\left(\chi_{\sigma}^{2}\right)_{\min }$ is the minimum of $\chi_{\sigma}^{2}$. This quantity can be used to test if a given point $\alpha$ in the parameter space is in agreement with the data set $\sigma$. For $\alpha$ we will use the best fit points from the global analysis for $(3+1)$ and $(2+2)$, the local minimum in $(2+2)$ and the point corresponding to the $(3+0)$ case. This approach is similar to the method proposed in Ref. [57].

Let us discuss the results of this analysis, which are shown in Tab. III. One can see that solar and atmospheric data are in perfect agreement with the global best fit point in $(3+1)$. The reason is that in this case both effects are explained by active neutrino oscillations, which is preferred by the data. Also a $\Delta \chi^{2}=3$ for LSND is in good agreement; the best fit point lies within the $90 \%$ C.L. region for the two parameters $\Delta m_{\mathrm{LSND}}^{2}$ and $\sin ^{2} 2 \theta_{\mathrm{LSND}}$. However, the $(3+1)$ best fit point gives a rather bad fit to the SBL experiments finding no evidence of oscillations: a value of $\Delta \chi^{2}=8.8$ for 2 d.o.f. $\left(d_{e}\right.$ and $\left.d_{\mu}\right)$ is ruled out at $98.7 \%$ C.L..

Regarding the $(2+2)$ scheme we observe some problems in the fit of solar or atmospheric data: At the best fit $(2+2)$ solution we obtain for solar data $\Delta \chi^{2}=10.7$ for 3 d.o.f., which is ruled out at $98.7 \%$ C.L., while the fit of the other data sets ATM, LSND and $\mathrm{NEV}$ is very good. The reason for the problems in the solar data is that the best fit for $(2+2)$ prefers a large value of $\eta_{s}$ (corresponding to the LOW solution). This implies a large component of the sterile neutrino in solar oscillations, which gives a bad fit. In the local minimum for $(2+2)$ - which is marked with a triangle in the upper right panel of Fig. 8 and corresponds to the LMA solution - the fit of solar data is very good, whereas atmospheric data gives a $\Delta \chi^{2}=11.5$ for 4 d.o.f., which is ruled out at $97.8 \%$ C.L.. In this case the bad fit is a consequence of the large sterile component in atmospheric oscillations implied by the small value of $\eta_{s}$. The interesting shape of $\chi_{\text {global }}^{2}\left(\eta_{s}\right)$ in $(2+2)$, which disfavors equal sterile admixture in solar and atmospheric oscillations, implies that either the solar or the atmospheric fit is bad in the $(2+2)$ case, but never both.

From the last column in Tab. III one can see that all experiments except LSND are in perfect agreement with the $(3+0)$ scenario. However, as expected the fit of LSND is very bad in this case and yields a $\Delta \chi^{2}=29$ for 2 d.o.f.. In the Gaussian approximation implied by Eq. (24) this would be ruled out at an extremely high C.L.. Let us note that far from 
the allowed region of LSND this approximation may not be completely justified. However, it is evident that LSND data is in strong disagreement with no oscillations. According to Table X of Ref. [11] the probability that the observed number of excess events is due to a fluctuation of the expected background is between $7.8 \times 10^{-6}$ and $1.8 \times 10^{-3}$, depending on different selection criteria applied to the data.

Some remarks are in order regarding this analysis for the NEV experiments. These experiments do not see any evidence for oscillations and hence, they obtain no information on a mass-squared difference; only an upper bound on the oscillation amplitude can be derived. Therefore, we consider $\Delta \chi_{\mathrm{NEV}}^{2}$ at a fixed value ${ }^{10}$ of $\Delta m_{\mathrm{LSND}}^{2}$. Hence, the $\Delta \chi^{2}$-values shown in the table have to be evaluated for 3 d.o.f. $\left(\theta_{\mathrm{LSND}}, d_{e}, d_{\mu}\right)$ in the $(2+2)$ scheme and for 2 d.o.f. for $(3+1)$ because of Eq. (11). Depending on the mass scheme we fix $\Delta m_{\mathrm{LSND}}^{2}$ at the best fit values given in Tab. I]; for $(3+0)$ we use the best fit value of $\Delta m_{\mathrm{LSND}}^{2}$ in the $(3+1)$ scheme. Although the NEV experiments are in agreement with no oscillations, the value $\Delta \chi_{\mathrm{NEV}}^{2}=2.3$ for $(3+0)$ shows that a small contribution of $\Delta m_{\mathrm{LSND}}^{2}$ can improve the fit slightly.

Table III confirms the results of Secs. VII and VIII. A combination of only solar and atmospheric data prefers the $(3+1)$ schemes. Therefore, these data are in perfect agreement with the global fit in $(3+1)$, but fit worse in $(2+2)$. On the other hand, atmospheric and SBL data prefer the $(2+2)$ scheme; the $(3+1)$ best fit point is somewhat in disagreement with NEV data. This conflict between different data sets does not show up in the $\chi^{2}$-values given in Eq. (45), since the coupling between the data sets is rather weak. As discussed in Sec. V1 only the parameter $\eta_{s}$ is common to solar and atmospheric oscillations, only the parameter $d_{\mu}$ links atmospheric and SBL data, while there is no direct coupling of solar and SBL data. All the other 7 parameters can be adjusted to give a good fit of the corresponding data set. The remaining conflict between the data sets is completely washed out by the large number of data points, which are fitted perfectly.

\section{CONCLUSIONS}

We have presented a unified global analysis of current neutrino oscillation data within the framework of four-neutrino mass schemes, paying attention to the inequivalent classes of $(3+1)$ and $(2+2)$ models. We have included all data from solar and atmospheric neutrino experiments, as well as information from short-baseline experiments including LSND and the null-result oscillation experiments. We have mapped the leptonic mixing matrix into a set of parameters in such a way that they have a well-defined physical meaning in each data set, independently of the mass scheme $((3+1)$ or $(2+2))$ considered. For example, one of these parameters is $\eta_{s}$, the fraction of sterile neutrinos in solar oscillations. Similarly $\theta_{24}$

${ }^{10}$ Note that the original analyses of the Bugey [36] and CDHS 38] collaborations were performed in this way. 
describes the fraction of $\nu_{\mu}$ in atmospheric oscillations and $\sin ^{2} 2 \theta_{\mathrm{LSND}}$ is characterizing the SBL $\stackrel{(-)}{\nu} \rightarrow \stackrel{(-)}{\nu} \nu_{e}$ amplitude. The fact that it is possible to describe the results of any of the given set of experiments in terms of physical quantities independent of the mass scheme implies that none of the considered data sets (solar, atmospheric, SBL appearance or SBL disappearance) can be used on its own to discriminate between different mass spectra. This follows from the approximation $\eta_{e} \approx 1$, which is motivated by the bounds from reactor neutrino experiments, and from the strong hierarchy among the mass-squared differences indicated by the data. We have shown how the differences between the mass schemes manifest themselves only when two or more data sets are combined.

We have found that combining only solar and atmospheric neutrino data, the $(3+1)$ type schemes are preferred, whereas atmospheric data in combination with short-baseline data prefers $(2+2)$ models. By combining all data in a global analysis the $(3+1)$ mass scheme gives a slightly better fit than the $(2+2)$ case, though all four-neutrino schemes are presently acceptable. The LSND result disfavors the three-active neutrino scenario with only $\Delta m_{\mathrm{SOL}}^{2}$ and $\Delta m_{\mathrm{ATM}}^{2}$ at $99.9 \%$ C.L. with respect to the four-neutrino best fit model. We have also performed a detailed analysis of the goodness of fit in order to identify which sub-set of the data is in disagreement with the best fit solution in a given mass scheme.

We have found that, in isolation, the LSND data play a crucial role in suggesting the need for a four-neutrino scenario, at odds with every other piece of information. The upcoming MiniBooNE experiment [59] will test the very important result of LSND in the near future. However, we have shown in this work that the existing data cannot decide between $(2+2)$ and $(3+1)$ mass schemes in a statistically significant way. Most likely this problem will remain also if MiniBooNE would confirm the LSND result, and to resolve the ambiguity more experimental information will be needed.

Such information could be provided by experiments with a high sensitivity to the sterile component in solar and/or atmospheric neutrino oscillations. One possibility to improve this sensitivity for atmospheric neutrinos could be the consideration of neutral current events in atmospheric neutrino experiments [60]. Concerning solar neutrinos, we note that the different oscillation solutions show very distinct behavior with respect to a sterile component (see Fig. 2); hence, the identification of the true solution is important. Moreover, improved measurements of neutral current event rates may increase the sensitivity to sterile oscillations. However, as shown in Ref. [61, the information obtainable from the neutral current measurements currently performed at SNO will be limited because of the relatively large uncertainty in the flux of solar ${ }^{8} \mathrm{~B}$ neutrinos. On the other hand, more data on $\stackrel{(-)}{\nu}$ and/or $\stackrel{(-)}{\nu}$ SBL disappearance probabilities could help to solve the $(3+1)$ versus $(2+2)$ puzzle. Especially the existing bounds on $\stackrel{(-)}{\nu}$ disappearance are rather weak. This will be improved by the MiniBooNE experiment, which - beside testing the $\nu_{\mu} \rightarrow \nu_{e}$ appearance channel will also provide a new measurement for the $\nu_{\mu}$ survival probability [59].

In view of the ambiguities implied by present data we are looking forward to the results of future neutrino oscillation experiments, which may unravel the secret behind the structure 
of the leptonic weak interaction.

\section{Acknowledgments}

We thank P. Huber and C. Peña-Garay for many useful discussions and W. Grimus for comments on a preliminary version of this paper. We are very grateful to W.C. Louis and G. Mills for providing us with the table of the LSND likelihood function. This work was supported by Spanish DGICYT under grant PB98-0693, by the European Commission RTN network HPRN-CT-2000-00148 and by the European Science Foundation network grant N. 86. T. S. was supported by a fellowship of the European Commission Research Training Site contract HPMT-2000-00124 of the host group. M. M. was supported by contract HPMFCT-2000-01008.

[1] Super-Kamiokande Coll., Y. Fukuda et al., Phys. Rev. Lett. 81, 1158 (1998); Erratum ibid. 81, 4279 (1998) and ibid. 82, 1810 and 2430 (1999); Y. Suzuki, Nucl. Phys. B (Proc. Suppl.) 77, 35 (1999); S. Fukuda et al., hep-ex/0103032.

[2] B.T. Cleveland et al., Astrophys. J. 496, 505 (1998); R. Davis, Prog. Part. Nucl. Phys. 32, 13 (1994); K. Lande, Talk given at Neutrino 2000, 15-21 June 2000, Sudbury, Canada [http://nu2000.sno.laurentian.ca].

[3] SAGE Coll., D.N. Abdurashitov et al., Phys. Rev. C60, 055801 (1999); V. Gavrin, Talk given at Neutrino 2000, 15-21 June 2000, Sudbury, Canada [http://nu2000.sno.laurentian.ca].

[4] GALleX Coll., W. Hampel et al., Phys. Lett. B447, 127 (1999); E. Belloti, GNO Coll., Talk given at Neutrino 2000, 15-21 June 2000, Sudbury, Canada [http://nu2000.sno.laurentian.ca].

[5] Q. R. Ahmad et al., SNO Coll., nucl-ex/0106015.

[6] Y. Fukuda et al., Kamiokande Coll., Phys. Lett. B 335 (1994) 237; R. Becker-Szendy et al., IMB Coll., Nucl. Phys. B (Proc. Suppl.) 38 (1995) 331; W.W.M. Allison et al., Soudan Coll., Phys. Lett. B 449 (1999) 137.

[7] Super-Kamiokande Coll., Y. Fukuda et al., Phys. Rev. Lett. 81 (1998) 1562; C. McGrew in Neutrino Telescopes 2001, Venice, Italy, March 2001, to appear; T. Toshito in Moriond 2001, Les Arcs, France, March 2001, to appear.

[8] MACRO Coll., M. Ambrosio et al., Phys. Lett. B 434 (1998) 451; M. Spurio et al., hepex/0101019; B. Barish, Talk given at Neutrino 2000, 15-21 June 2000, Sudbury, Canada [http://nu2000.sno.laurentian.ca].

[9] A. Surdo, Talk given at TAUP 2001, 8-12 September 2001, Gran Sasso, Italy [http://www.lngs.infn.it/]. 
[10] C. Athanassopoulos et al., LSND Coll., Phys. Rev. Lett. 77 (1996) 3082; ibid 81 (1998) 1774; G. Mills, LSND Coll., Talk given at Neutrino 2000, 15-21 June 2000, Sudbury, Canada [http://nu2000.sno.laurentian.ca].

[11] A. Aguilar et al., LSND Coll., hep-ex/0104049.

[12] H. Murayama and T. Yanagida, hep-ph/0010178; G. Barenboim et al., hep-ph/0108199.

[13] J. T. Peltoniemi, D. Tommasini and J. W. F. Valle, Phys. Lett. B 298 (1993) 383.

[14] J. T. Peltoniemi and J. W. F. Valle, Nucl. Phys. B 406, 409 (1993) hep-ph/9302316.

[15] D.O. Caldwell and R.N. Mohapatra, Phys. Rev. D 48 (1993) 3259.

[16] E. Ma and P. Roy, Phys. Rev. D 52 (1995) R4780; E.J. Chun et al., Phys. Lett. B 357 (1995) 608; J.J. Gomez-Cadenas and M.C. Gonzalez-Garcia, Z. Phys. C 71 (1996) 443; E. Ma, Mod. Phys. Lett. A 11 (1996) 1893; S. Goswami, Phys. Rev. D 55 (1997) 2931; Q.Y. Liu and A. Smirnov, Nucl. Phys. B 524 505; V. Barger, K. Whisnant and T. Weiler, Phys. Lett. B 427 (1998) 97; S. Gibbons, R.N. Mohapatra, S. Nandi and A. Raichoudhuri, Phys. Lett. B 430 (1998) 296; Nucl. Phys. B 524 (1998) 505; E.J. Chun, A. Joshipura and A. Smirnov, in Elementary Particle Physics: Present and Future (World Scientific, 1996), ISBN 981-02-2554-7; P. Langacker, Phys. Rev. D 58 (1998) 093017; M. Bando and K. Yoshioka, Prog. Theor. Phys. 100 (1998) 1239; W. Grimus, R. Pfeiffer and T. Schwetz, Eur. Phys. J. C 13 (2000) 125; F. Borzumati, K. Hamaguchi and T. Yanagida, Phys. Lett. B 497 (2001) 259; K. R. Balaji, A. Perez-Lorenzana and A. Y. Smirnov, Phys. Lett. B 509 (2001) 111; K.S. Babu and R.N. Mohapatra, hep-ph/0110243; S. Goswami and A.S. Joshipura, hep-ph/0110272.

[17] R. N. Mohapatra, A. Perez-Lorenzana and C. A. de S Pires, Phys. Lett. B 491 (2000) 14.

[18] A. Ioannisian and J. W. F. Valle, Phys. Rev. D 63 (2001) 073002; hep-ph/9911349.

[19] M. Hirsch and J. W. F. Valle, Phys. Lett. B 495 (2000) 121 hep-ph/0009066.

[20] Web-page of C. Giunti, http://www.to.infn.it/ giunti/neutrino/ .

[21] V. Barger et al., Phys. Lett. B 489 (2000) 345.

[22] O.L.G. Peres and A.Yu. Smirnov, Nucl. Phys. B 599 (2001) 3.

[23] N. Okada and O. Yasuda, Int. J. Mod. Phys. A 12 (1997) 3669.

[24] V. Barger, S. Pakvasa, T.J. Weiler and K. Whisnant, Phys. Rev. D 58 (1998) 093016.

[25] S.M. Bilenky, C. Giunti, W. Grimus and T. Schwetz, Phys. Rev. D 60 (1999) 073007.

[26] S.M. Bilenky, C. Giunti and W. Grimus, in Proceedings of Neutrino '96, Helsinki, Finland, 13-19 June 1996, p. 174, edited by K. Enqvist, K. Huitu and J. Maalampi (World Scientific, Singapore 1997); S.M. Bilenky, C. Giunti and W. Grimus, Eur. Phys. J. C 1 (1998) 247.

[27] C. Giunti and M. Laveder, JHEP 0102 (2001) 001.

[28] W. Grimus and T. Schwetz, Eur. Phys. J. C 20 (2001) 1 hep-ph/0102252.

[29] M. Maltoni, T. Schwetz and J. W. F. Valle, Phys. Lett. B 518, 252 (2001) hep-ph/0107150.

[30] S. Fukuda et al., Super-Kamiokande Coll., hep-ex/0103033; Phys. Rev. Lett. 85, 3999 (2000).

[31] J. N. Bahcall, M. C. Gonzalez-Garcia and C. Pena-Garay, hep-ph/0106258.

[32] M.C. Gonzalez-Garcia, M. Maltoni and C. Peña-Garay, Phys. Rev. D 64 (2001) 093001 [hep$\mathrm{ph} / 0105269]$. 
[33] M. C. Gonzalez-Garcia, M. Maltoni and C. Peña-Garay, hep-ph/0108073.

[34] K. Eitel and B. Zeitnitz, KARMEN Coll., in Proceedings of Neutrino '98, Takayama, Japan, 4-9 June 1998, Nucl. Phys. Proc. Suppl. 77, 212 (1999); K. Eitel, KARMEN Coll., Talk given at Neutrino 2000, 15-21 June 2000, Sudbury, Canada, hep-ex/0008002.

[35] M. Mezzetto, NOMAD Coll., Nucl. Phys. B (Proc. Suppl.) 70 (1999) 214; L. Camilleri, NOMAD Coll., A Status Report (1999) [http://nomadinfo.cern.ch/Public/PUBLICATIONS/].

[36] B. Achkar et al., Bugey Coll., Nucl. Phys. B 434 (1995) 503.

[37] M. Apollonio et al., CHOOZ Coll., Phys. Lett. B 466 (1999) 415.

[38] F. Dydak et al., CDHS Coll., Phys. Lett. B 134 (1984) 281.

[39] J. Schechter and J. W. F. Valle, Phys. Rev. D 22 (1980) 2227.

[40] J. Schechter and J. W. F. Valle, Phys. Rev. D 23 (1981) 1666.

[41] S.M. Bilenky, C. Giunti and W. Grimus, Phys. Rev. D 58 (1998) 033001; V. Barger et al., Phys. Rev. D 59 (1999) 113010; K. Dick et al., Nucl. Phys. B 562 (1999) 29; A. Donini et al., Nucl. Phys. B 574 (2000) 23; A. Kalliomaki, J. Maalampi and M. Tanimoto, Phys. Lett. B 469 (1999) 179; T. Hattori, T. Hasuike and S. Wakaizumi, Phys. Rev. D 62 (2000) 033006;

A. Donini and D. Meloni, hep-ph/0105089.

[42] Y. Farzan, O.L.G. Peres and A.Yu. Smirnov, Nucl. Phys. B 621 (2001) 59 hep-ph/0105105.

[43] S.M. Bilenky, S. Pascoli and S.T. Petcov, hep-ph/0104218; A. Kalliomaki and J. Maalampi, Phys. Lett. B 484 (2000) 64 [hep-ph/0003281]; S. M. Bilenky, C. Giunti, W. Grimus, B. Kayser and S. T. Petcov, Phys. Lett. B 465 (1999) 193 [hep-ph/9907234.

[44] F. Boehm et al., Palo Verde Coll., Phys. Rev. D 64 (2001) 112001 hep-ex/0107009.

[45] M. C. Gonzalez-Garcia, P. C. de Holanda, C. Pena-Garay and J. W. F. Valle, Nucl. Phys. B 573 (2000) 3 hep-ph/9906469.

[46] W.C. Louis and G. Mills, LSND Coll., private communication.

[47] D.E. Groom et al., Review of Particle Physics, Eur. Phys. J. C 15 (2000) 1.

[48] D. Dooling, C. Giunti, K. Kang and C. W. Kim, Phys. Rev. D 61 (2000) 073011 [hep$\mathrm{ph} / 9908513$.

[49] C. Giunti, M. C. Gonzalez-Garcia and C. Peña-Garay, Phys. Rev. D 62 (2000) 013005 [hep$\mathrm{ph} / 0001101$.

[50] S. M. Bilenky, C. Giunti, W. Grimus and T. Schwetz, Astropart. Phys. 11 (1999) 413 [hep$\mathrm{ph} / 9804421$.

[51] M. C. Gonzalez-Garcia, M. Maltoni, C. Pena-Garay and J. W. F. Valle, Phys. Rev. D 63 (2001) 033005 hep-ph/0009350; N. Fornengo, M.C. Gonzalez-Garcia and J. W. F. Valle, Nucl. Phys. B 580 (2000) 58; M. C. Gonzalez-Garcia, H. Nunokawa, O. L. G. Peres and J. W. F. Valle, Nucl. Phys. B 543 (1999) 3 [hep-ph/9807305].

[52] G. L. Fogli, E. Lisi and A. Marrone, Phys. Rev. D 63 (2001) 053008 hep-ph/0009299; O. Yasuda, hep-ph/0006319.

[53] K. Eitel, New Jour. Phys. 2 (2000) 1 [hep-ex/9909036].

[54] L. Borodovsky et al., Phys. Rev. Lett. 68 (1992) 274. 
[55] A. Romosan et al., Phys. Rev. Lett. 78 (1997) 2912; I.E. Stockdale et al., Phys. Rev. Lett. 52 (1984) 1384; Z. Phys. C 53 (1985) 53.

[56] J. N. Bahcall, M. C. Gonzalez-Garcia and C. Pena-Garay, hep-ph/0111150.

[57] J. C. Collins and J. Pumplin, hep-ph/0105207.

[58] P. Creminelli, G. Signorelli and A. Strumia, JHEP 0105 (2001) 052 hep-ph/0102234;

M. V. Garzelli and C. Giunti, hep-ph/0108191; P.I. Krastev and A.Yu. Smirnov, hep$\mathrm{ph} / 0108177$.

[59] A. Bazarko, MiniBooNE Coll., Talk given at Neutrino 2000, 15-21 June 2000, Sudbury, Canada [http://nu2000.sno.laurentian.ca].

[60] A. Habig, Super-Kamiokande Coll., hep-ex/0106025.

[61] V. D. Barger, D. Marfatia and K. Whisnant, Phys. Rev. Lett. 88 (2002) 011302 [hep$\mathrm{ph} / 0106207$. 